\title{
The essentially tame local Langlands correspondence, II: totally ramified representations
}

\author{
Colin J. Bushnell and Guy Henniart
}

\begin{abstract}
Let $F$ be a non-Archimedean local field. Let $\mathcal{G}_{n}^{\text {et }}(F)$ be the set of equivalence classes of irreducible, $n$-dimensional representations of the Weil group $\mathcal{W}_{F}$ of $F$ which are essentially tame. Let $\mathcal{A}_{n}^{\text {et }}(F)$ be the set of equivalence classes of irreducible, essentially tame, supercuspidal representations of $\mathrm{GL}_{n}(F)$. The Langlands correspondence induces a canonical bijection $\mathcal{L}: \mathcal{G}_{n}^{\text {et }}(F) \rightarrow \mathcal{A}_{n}^{\text {et }}(F)$. We continue the programme of describing this map in terms of explicit descriptions of the sets $\mathcal{G}_{n}^{\text {et }}(F)$ and $\mathcal{A}_{n}^{\text {et }}(F)$. These descriptions are in terms of admissible pairs $(E / F, \xi)$, consisting of a tamely ramified field extension $E / F$ of degree $n$ and a quasicharacter $\xi$ of $E^{\times}$subject to certain technical conditions. If $P_{n}(F)$ is the set of isomorphism classes of admissible pairs of degree $n$, we have explicit bijections $P_{n}(F) \cong \mathcal{G}_{n}^{\text {et }}(F)$ and $P_{n}(F) \cong \mathcal{A}_{n}^{\text {et }}(F)$. In an earlier paper we showed that, if $\sigma \in \mathcal{G}_{n}^{\text {et }}(F)$ corresponds to an admissible pair $(E / F, \xi)$, then $\mathcal{L}(\sigma)$ corresponds to the admissible pair $(E / F, \mu \xi)$, for a certain tamely ramified character $\mu$ of $E^{\times}$. In this paper, we determine the character $\mu$ when $E / F$ is totally ramified.
\end{abstract}

\section{Introduction}

This paper, written in continuation of [BH05a], gives the next step towards an explicit description of the local Langlands correspondence for essentially tame representations. Throughout, $F$ is a non-Archimedean local field, the residue field of which is finite and of characteristic $p$.

An irreducible smooth representation $\sigma$ of the Weil group $\mathcal{W}_{F}$ of $F$, of dimension $n$, is essentially tame if the number $t(\sigma)$ of unramified characters $\chi$ of $\mathcal{W}_{F}$, such that $\chi \otimes \sigma \cong \sigma$, satisfies $p \nmid n / t(\sigma)$. We write $\mathcal{G}_{n}^{\text {et }}(F)$ for the set of equivalence classes of $n$-dimensional, irreducible, essentially tame representations of $\mathcal{W}_{F}$. Likewise, if $\pi$ is an irreducible supercuspidal representation of $\mathrm{GL}_{n}(F)$, we define $t(\pi)$ to be the number of unramified characters $\chi$ of $F^{\times}$such that $\pi$ is equivalent to the representation $\chi \pi: g \mapsto \chi(\operatorname{det} g) \pi(g)$. We say that $\pi$ is essentially tame if $p \nmid n / t(\pi)$, and we denote the set of equivalence classes of such representations by $\mathcal{A}_{n}^{\text {et }}(F)$. The Langlands correspondence [LRS93, HT01, Hen00] induces a bijection

$$
\mathcal{L}={ }_{F} \mathcal{L}_{n}^{\mathrm{et}}: \mathcal{G}_{n}^{\mathrm{et}}(F) \stackrel{\approx}{\underset{ }{\longrightarrow}} \mathcal{A}_{n}^{\mathrm{et}}(F) .
$$

It is this map we wish to describe.

There is an explicit bijection ${ }_{F} \mathcal{N}_{n}: \mathcal{G}_{n}^{\text {et }}(F) \rightarrow \mathcal{A}_{n}^{\text {et }}(F)\left[\right.$ BH05a] with which we can compare ${ }_{F} \mathcal{L}_{n}^{\text {et }}$. Given $\sigma \in \mathcal{G}_{n}^{\text {et }}(F)$, there is a tamely ramified field extension $E / F$ and a quasicharacter $\xi$ of $E^{\times}$

Received 18 March 2004, accepted in final form 23 September 2004, published online 21 June 2005.

2000 Mathematics Subject Classification 22E50.

Keywords: explicit local Langlands correspondence, base change, automorphic induction, tame lifting, admissible pair.

Much of the work in this programme was carried out while the first-named author was visiting, and partly supported by, l'Université de Paris-Sud. The first-named author wishes to thank King's College London for hospitality during the course of this work. Both authors were also partially supported by EU network 'Arithmetical Algebraic Geometry'. This journal is (c) Foundation Compositio Mathematica 2005. 


\section{J. Bushnell and G. Henniart}

such that $\sigma \cong \operatorname{Ind}_{E / F} \xi$. Here, we regard $\xi$ as a quasicharacter of $\mathcal{W}_{E}$ via local class field theory, and $\operatorname{Ind}_{E / F}$ denotes the operation of induction from $\mathcal{W}_{E}$ to $\mathcal{W}_{F}$. One can refine this: one imposes a condition of 'admissibility' on the pair $(E / F, \xi)$ (we recall the definition below) to obtain a canonical bijection

$$
\begin{aligned}
P_{n}(F) & \longrightarrow \mathcal{G}_{n}^{\text {et }}(F), \\
(E / F, \xi) & \longmapsto \operatorname{Ind}_{E / F} \xi,
\end{aligned}
$$

between $\mathcal{G}_{n}^{\text {et }}(F)$ and the set $P_{n}(F)$ of $F$-isomorphism classes of admissible pairs $(E / F, \xi)$ in which $[E: F]=n$. Using the classification theory for supercuspidal representations [BK93], we constructed in $[\mathrm{BH} 05 \mathrm{a}]$ a canonical, and explicit, bijection

$$
\begin{aligned}
P_{n}(F) & \longrightarrow \mathcal{A}_{n}^{\mathrm{et}}(F), \\
(E / F, \xi) & \longmapsto{ }_{F} \pi_{\xi},
\end{aligned}
$$

of which we recall relevant details below. The bijection ${ }_{F} \mathcal{N}_{n}: \mathcal{G}_{n}^{\text {et }}(F) \rightarrow \mathcal{A}_{n}^{\text {et }}(F)$ is then that induced by (1) and (2).

If $K / F$ is a finite field extension and $1_{K}$ denotes the trivial representation of $\mathcal{W}_{K}$, we write $\delta_{K / F}=\operatorname{det} \operatorname{Ind}_{K / F} 1_{K}$. The bijections ${ }_{F} \mathcal{L}_{n}^{\text {et }}$ and ${ }_{F} \mathcal{N}_{n}$ are related as follows [BH05a, (3.3)].

Theorem A. Let $(E / F, \xi) \in P_{n}(F)$ and set $\sigma=\operatorname{Ind}_{E / F} \xi$. There exists a tamely ramified character $\mu={ }_{F} \mu_{\xi}$ of $E^{\times}$such that $\mathcal{L}(\sigma)={ }_{F} \pi_{\mu \xi}$.

To describe $\mathcal{L}={ }_{F} \mathcal{L}_{n}^{\text {et }}$ therefore, we have to determine the character ${ }_{F} \mu_{\xi}$. In [BH05a, (4.4)], we dealt with a first case as follows.

Theorem B. Suppose $n$ is odd, let $(E / F, \xi) \in P_{n}(F)$ and suppose that $E / F$ is totally ramified. Then

$$
{ }_{F} \mu_{\xi}=\delta_{E / F} \circ \mathrm{N}_{E / F}
$$

In the present paper, we calculate $F \mu_{\xi}$ when $(E / F, \xi) \in P_{n}(F)$ has $E / F$ totally ramified and $n$ is arbitrary: general admissible pairs $(E / F, \xi)$ will be treated in the next paper of the series. We assume that $n$ is even, and we let $K / F$ be the unique quadratic sub-extension of $E / F$. We set $\rho={ }_{K} \pi_{\xi} \in \mathcal{A}_{n / 2}^{\text {et }}(K)$, and we let $\pi=\mathrm{A}_{K / F} \rho \in \mathcal{A}_{n}^{\text {et }}(F)$ be the representation that is automorphically induced by $\rho$ (see [HH95, HL05]). There is a tamely ramified character $\mu={ }_{K / F} \mu_{\xi}$ of $E^{\times}$such that $(E / F, \mu \xi) \in P_{n}(F)$ and $\pi={ }_{F} \pi_{\mu \xi}$ (see [BH05a, (3.4)]). A key step is then given by Corollary 3.3 below:

$$
{ }_{F} \mu_{\xi}=K / F \mu_{\xi} \cdot K \mu_{\xi}
$$

Inductively, we can assume that $K \mu_{\xi}$ is known, and we have to determine $K_{K / F} \mu_{\xi}$.

To do this, we use the automorphic induction equation. We view $E$ as embedded in $\mathrm{M}_{n}(F)$ and we set $\Sigma=\operatorname{Gal}(K / F)$. We then have the character relation [HH95, HL05]

$$
\Xi_{\pi}^{\varkappa}(h)=c \boldsymbol{\delta}(h) \sum_{\sigma \in \Sigma} \operatorname{tr} \rho^{\sigma}(h),
$$

valid for all elliptic regular elements $h$ of $\mathrm{GL}_{n}(F)$ which commute with $K$. Here, $\boldsymbol{\delta}(h)$ is the transfer factor $\Delta^{2}(h) / \Delta^{1}(h)$ of [HH95] and $c$ is a certain non-zero constant (of which nothing is known $a$ priori). The function $\Xi_{\pi}^{\varkappa}$ is the $\varkappa$-twisted trace of $\pi$, formed relative to the non-trivial character $\varkappa$ of $F^{\times}$vanishing on norms from $K^{\times}$.

By evaluating both sides of (3) at prime elements $\varpi$ of $E$, we get the following theorem [BH05a, $(4.3)]$. 


\section{TAME LANGLANDS CORRESPONDENCE: TOTALLY RAMIFIED REPRESENTATIONS}

Theorem C. The tamely ramified character ${ }_{K / F} \mu_{\xi}$ satisfies ${ }_{K / F} \mu_{\xi} \mid F^{\times}=\delta_{K / F}^{n / 2}$ and, for every prime element $\varpi$ of $E$,

$$
K / F \mu_{\xi}(\varpi)=\epsilon_{F} \epsilon_{K} c \boldsymbol{\delta}(\varpi)
$$

Here, $\epsilon_{F}$ and $\epsilon_{K}$ are constants, with values \pm 1 , determined by details of the internal structure of $(E / F, \xi)$ and $(E / K, \xi)$, respectively. (We recall the definition below, in 'Background'.) The major step in this paper calculates both sides of (3) at elements $h=1+\varpi$, where, again, $\varpi$ is a prime element of $E$. This gives an expression for $c \boldsymbol{\delta}(\varpi)$ in terms of quadratic Gauss sums and Kloosterman sums. (The arguments and results in this part hold in greater generality.)

Some labour is then required to evaluate these trigonometric sums and the sign $\epsilon_{F} \epsilon_{K}$. The final form of ${ }_{F} \mu_{\xi}$ is given as Theorem 2.1. The character ${ }_{F} \mu_{\xi}$ has order dividing 4 . The formula for its values is quite complex and depends, with a considerable degree of sensitivity, on the internal structure of $\xi$. The result is not the same as that predicted conjecturally by [Rei91] (which builds on [How77], [Moy86] and [BF83]). In $\S 2.2$, we discuss briefly the approach of [Rei91] and give an example illustrative of the discrepancy between the predictions of that paper and the reality established here.

We have also included a result (§ 2.3) describing the (surprisingly simple) behaviour of the character ${ }_{F} \mu_{\xi}$ with respect to unramified base change. This generalizes Theorem 4.6 of [BH05a] which is, however, used in its proof.

\section{Notation}

Throughout, $F$ is a non-Archimedean local field; the discrete valuation ring in $F$ is $\mathfrak{o}_{F}$, the maximal ideal of $\mathfrak{o}_{F}$ is $\mathfrak{p}_{F}$, while the residue field $\mathbb{k}_{F}=\mathfrak{o}_{F} / \mathfrak{p}_{F}$ has $q$ elements and characteristic $p$. We write $U_{F}=\mathfrak{o}_{F}^{\times}$and $U_{F}^{n}=1+\mathfrak{p}_{F}^{n}, n \geqslant 1$. Thus $U_{F}=\boldsymbol{\mu}_{F} \times U_{F}^{1}$, where $\boldsymbol{\mu}_{F}$ is the group of roots of unity in $F$ of order prime to $p$. We let $v_{F}$ be the normalized additive valuation $F^{\times} \rightarrow \mathbb{Z}$.

We use analogous notation relative to a finite extension field $E / F$; we denote by $\mathrm{N}_{E / F}$ and $\operatorname{Tr}_{E / F}$ the relative norm and trace, respectively.

If $V$ is a finite-dimensional $F$-vector space and $\mathfrak{A}$ is a hereditary $\mathfrak{o}_{F}$-order in $A=\operatorname{End}_{F}(V)$, we write $U_{\mathfrak{A}}=\mathfrak{A}^{\times}$and $U_{\mathfrak{A}}^{n}=1+\mathfrak{P}^{n}, n \geqslant 1$, where $\mathfrak{P}$ is the Jacobson radical of $\mathfrak{A}$. We write $\mathcal{K}_{\mathfrak{A}}$ for the group of $x \in G=\operatorname{Aut}_{F}(V)$ such that $x^{-1} \mathfrak{A} x=\mathfrak{A}$.

If $[\mathfrak{A}, l, 0, \beta]$ is a simple stratum in $A$, we define compact open subgroups $H^{m}(\beta, \mathfrak{A}), m \geqslant 1$, and $J^{n}(\beta, \mathfrak{A}), n \geqslant 0$, of $G$ as in [BK93, ch. 3]. Let $\psi_{F}$ be a character of $F$, trivial on $\mathfrak{p}_{F}$ but non-trivial on $\mathfrak{o}_{F}$. As in [BK93], $\mathcal{C}\left(\mathfrak{A}, \beta, \psi_{F}\right)$ is the set of simple characters of $H^{1}(\beta, \mathfrak{A})$ attached to $[\mathfrak{A}, l, 0, \beta]$ and $\psi_{F}$.

\section{Background}

For convenience, we recall some of the basic ideas of [BH05a]. First, consider a pair $(E / F, \xi)$ consisting of a finite, tamely ramified field extension $E / F$ and a quasicharacter $\xi$ of $E^{\times}$. Let $K$ range over the fields such that $F \subset K \subset E$. We say that $(E / F, \xi)$ is admissible if $\xi$ does not factor through the norm $\mathrm{N}_{E / K}$ when $K \neq E$, while if $\xi \mid U_{E}^{1}$ factors through $\mathrm{N}_{E / K}$, then $E / K$ is unramified. We write $P_{n}(F)$ for the set of $F$-isomorphism classes of admissible pairs $(E / F, \xi)$ such that $[E: F]=n$.

We recall briefly the definition [BH05a, $\S \S 2.3$ and 4.1] of the representation ${ }_{F} \pi_{\xi} \in \mathcal{A}_{n}^{\text {et }}(F)$ when $(E / F, \xi) \in P_{n}(F)$ and $E / F$ is totally ramified. We view $E$ as embedded in $A=\mathrm{M}_{n}(F)$; there is then a unique hereditary $\mathfrak{o}_{F}$-order $\mathfrak{A}$ in $A$ such that $E^{\times} \subset \mathcal{K}_{\mathfrak{A}}$. There is a simple stratum $[\mathfrak{A}, l, 0, \beta]$ in $A$, such that $E=F[\beta]$, and a simple character $\theta \in \mathcal{C}\left(\mathfrak{A}, \beta, \psi_{F}\right)$, such that $\theta\left|U_{E}^{1}=\xi\right| U_{E}^{1}$. We write $H^{1}=H^{1}(\beta, \mathfrak{A}), J^{1}=J^{1}(\beta, \mathfrak{A}), J^{0}=J(\beta, \mathfrak{A})=\boldsymbol{\mu}_{F} J^{1}$ and $\boldsymbol{J}=E^{\times} J^{1}$. Let $\eta$ denote the unique irreducible representation of $J^{1}$ such that $\eta \mid H^{1}$ is a multiple of $\theta$. 


\section{J. Bushnell and G. Henniart}

Consider the finite $p$-group $\mathcal{J}=J^{1} / \operatorname{Ker} \theta$. The group $E^{\times}$acts on this group by conjugation, and the subgroup $F^{\times} U_{E}^{1}$ acts trivially. Write $\Gamma=E^{\times} / F^{\times} U_{E}^{1}$, so that $\Gamma$ is cyclic of order $n$. There is then a unique irreducible representation $\tilde{\eta}$ of the semi-direct product $\Gamma \ltimes \mathcal{J}$ such that $\tilde{\eta} \mid \mathcal{J} \cong \eta$ and $\operatorname{det} \tilde{\eta} \mid \Gamma=1$. There is a constant $\epsilon_{F}= \pm 1$ such that $\operatorname{tr} \tilde{\eta}(\gamma)=\epsilon_{F}$, for every generator $\gamma$ of $\Gamma$. Finally, there is a unique irreducible representation $\Lambda$ of $\boldsymbol{J}$ such that

$$
\Lambda \mid J^{1} \cong \eta \text { and } \operatorname{tr} \Lambda(x)=\epsilon_{F} \xi(x),
$$

for every $x \in E^{\times}$such that $v_{E}(x)$ is prime to $n$. We then have

$$
{ }_{F} \pi_{\xi}=c-\operatorname{Ind}_{J}^{G} \Lambda
$$

where $G=\mathrm{GL}_{n}(F)$.

\section{Invariants of admissible pairs}

We take an admissible pair $(E / F, \xi) \in P_{n}(F)$ in which $E / F$ is totally ramified: in particular, $p \nmid n$. In order to state our main results, we have to define certain invariants of the pair $(E / F, \xi)$. These will only depend on the restriction $\xi \mid U_{E}^{1}$.

We also recall the definition and elementary properties of the Langlands constant $\lambda_{E / F}$ attached to the extension $E / F$, since it allows us to express our results more neatly.

Throughout, we work relative to a fixed character $\psi_{F}$ of $F$ which is of level one: thus $\psi_{F}$ is trivial on $\mathfrak{p}_{F}$ but not on $\mathfrak{o}_{F}$. If $K / F$ is a finite, tamely ramified field extension, we set $\psi_{K}=\psi_{F} \circ \operatorname{Tr}_{K / F}$. Thus $\psi_{K}$ is a character of $K$ of level one.

1.1 Let $l$ be the greatest integer such that $\xi \mid U_{E}^{l}$ is non-trivial. For each integer $i \geqslant 0$, there is a unique subfield $E_{i} / F$ such that $\xi \mid U_{F}^{i+1}$ factors through $\mathrm{N}_{E / E_{i}}$, and which is minimal for this property [BH05a, (A.1)]. Thus $E_{0}=E$ while $E_{i}=F$ for $i \geqslant l+1$.

We put $d_{i}=\left[E_{i}: F\right]$. We say that $i \geqslant 1$ is a jump of $(E / F, \xi)$ if $d_{i} \neq d_{i-1}$. We denote by $S(\xi)$ the set of jumps of $\xi$.

Let $\alpha \in E$, and suppose that $v_{E}(\alpha)=-i<0$. Let $j$ be an integer, $j<i+1 \leqslant 2 j$. We define a character $\psi_{E, \alpha}$ of $U_{E}^{j}$ by

$$
\psi_{E, \alpha}: 1+x \longmapsto \psi_{E}(\alpha x), \quad x \in \mathfrak{p}_{E}^{j} .
$$

Let $i, 1 \leqslant i \leqslant l$, be a jump of $\xi$, and consider $\xi \mid U_{E}^{1}$. We may write

$$
\xi \mid U_{E}^{1}=\phi_{i} \otimes \chi_{i} \circ \mathrm{N}_{E / E_{i}}
$$

for a character $\chi_{i}$ of $U_{E_{i}}^{1}$ and a non-trivial character $\phi_{i}$ which is trivial on $U_{E}^{i+1}$. Since $\phi_{i} \mid U_{E}^{i}$ factors through $\mathrm{N}_{E / E_{i-1}}$, we may write

$$
\phi_{i} \mid U_{E}^{i}=\psi_{E, \alpha_{i}}
$$

for some $\alpha_{i} \in E_{i-1}$ with $v_{E}\left(\alpha_{i}\right)=-i$. The coset $\alpha_{i}+\mathfrak{p}_{E_{i}}^{1-i}=\alpha_{i} U_{E_{i}}^{1}$ is uniquely determined by $\phi_{i}$ and, by definition, we have $E_{i-1}=E_{i}\left[\alpha^{\prime}\right]$, for any $\alpha^{\prime} \in \alpha_{i} U_{E_{i-1}}^{1}$.

\section{Proposition 1.1.}

(1) We have $d_{i-1} / d_{i}=n / \operatorname{gcd}\left(i d_{i}, n\right)$.

(2) The coset $\alpha_{i}+\mathfrak{p}_{E}^{1-i}$ does not depend on the choice of character $\chi_{i}$.

Proof. The coset $\alpha_{i} U_{E_{i-1}}^{1}$ has the property that $E_{i-1}=E_{i}\left[\alpha^{\prime}\right]$, for any $\alpha^{\prime} \in \alpha_{i} U_{E_{i-1}}^{1}$. Since $E_{i-1} / E_{i}$ is totally tamely ramified, this is equivalent to $v_{E_{i-1}}\left(\alpha_{i}\right)$ being relatively prime to $d_{i-1} / d_{i}=$ $e\left(E_{i-1} \mid E_{i}\right)$. This gives part (1), and part (2) follows since the coset $\alpha_{i} U_{E}^{1}$ contains no element of $E_{i}$. 


\section{TAME LANGLANDS CORRESPONDENCE: TOTALLY RAMIFIED REPRESENTATIONS}

Note, however, that $\alpha_{i} U_{E}^{1}$ does depend on the choice of $\psi_{F}$.

1.2 We suppose now that $n=[E: F]$ is even (so, in particular, $p \neq 2$ ). Let $i$ be the least jump of $\xi$ such that $n / d_{i}=\left[E: E_{i}\right]$ is even. Thus the integer $\left[E: E_{i-1}\right]=n / d_{i-1}$ is odd; according to Proposition 1.1, part (1), $n / d_{i-1}=\left(i, n / d_{i}\right)$, whence $i$ is odd. Every jump $j>i$ is divisible by $n / d_{i}$ and so is even. We conclude that $i$ is the largest odd jump of $\xi$. It will be useful to have some standard notation

Notation. Suppose that $n=[E: F]$ is even. Define:

(1) $\boldsymbol{i}^{+}=$the largest odd jump of $\xi$;

(2) $\boldsymbol{d}^{+}=d_{\boldsymbol{i}^{+}}=\left[E_{\boldsymbol{i}^{+}}: F\right]$;

(3) $\boldsymbol{i}_{+}=$the least jump $j$ of $\xi$ such that $d_{j}$ is odd

Summarizing the definitions and the preceding remarks, we have the following lemma.

\section{LEMMA 1.2 .}

(1) We have $1 \leqslant \boldsymbol{i}^{+} \leqslant \boldsymbol{i}_{+} \leqslant l$; moreover, $\boldsymbol{i}^{+}=\boldsymbol{i}_{+}$if and only if $\boldsymbol{i}_{+}$is odd.

(2) Let $K_{2} / F$ be the largest sub-extension of $E / F$ such that $\left[K_{2}: F\right]$ is a power of 2 . Then

(a) $\boldsymbol{i}^{+}$is the largest jump $i$ of $\xi$ such that $K_{2} \subset E_{i-1}$;

(b) $\boldsymbol{i}_{+}$is the least jump $j$ of $\xi$ such that $K_{2} \cap E_{j}=F$.

1.3 We consider the element $\alpha=\alpha(\xi)=\alpha_{\boldsymbol{i}^{+}}$, in the notation of $\S 1.1$. We recall that only the coset $\alpha(\xi) U_{E}^{1}$ is well defined.

Let $\varpi$ be a prime element of $E$. Since $v_{E}(\alpha)=-\boldsymbol{i}^{+}$, there is a unique root of unity $\zeta(\varpi, \xi) \in \boldsymbol{\mu}_{F}$ such that

$$
\zeta(\varpi, \xi) \equiv \varpi^{i^{+}} \alpha(\xi) \quad\left(\bmod U_{E}^{1}\right)
$$

Remark 1.3. Suppose we have a subfield $F_{0}$ of $F$ such that the extension $F / F_{0}$ is ramified quadratic, and such that the pair $\left(E / F_{0}, \xi\right)$ is admissible. Replacing $F$ by $F_{0}$ then does not affect the jump $\boldsymbol{i}^{+}$ or the root of unity $\zeta(\varpi, \xi)$, but it may change $\boldsymbol{i}_{+}$and $\boldsymbol{d}^{+}$.

1.4 We write $q=\left|\mathbb{k}_{F}\right|$, and use the Legendre symbol

$$
x \longmapsto\left(\frac{x}{q}\right), \quad x \in \mathbb{k}_{F}^{\times},
$$

to denote the quadratic character of $\mathbb{k}_{F}^{\times}$. We regard this equally as a character of $\boldsymbol{\mu}_{F}$.

If $\psi$ is a non-trivial character of $\mathbb{k}_{F}$, we may form the classical Gauss sum

$$
\mathfrak{g}(\psi)=\sum_{x \in \mathbb{R}_{F}^{\times}}\left(\frac{x}{q}\right) \psi(x) .
$$

This is a complex number of absolute value $q^{1 / 2}$. It is useful to have a notation for the normalized version:

$$
\mathfrak{w}(\psi)=q^{-1 / 2} \mathfrak{g}(\psi) .
$$

Our character $\psi_{F}$ defines, by reduction, a non-trivial character $\psi_{F}^{0}$ of $\mathbb{k}_{F}$; we write $\mathfrak{w}\left(\psi_{F}\right)=\mathfrak{w}\left(\psi_{F}^{0}\right)$. Thus $\mathfrak{w}\left(\psi_{F}\right)$ is a fourth root of unity in $\mathbb{C}$. 


\section{J. Bushnell and G. Henniart}

1.5 Suppose, for the moment, that $\sigma$ is a finite-dimensional, $\Phi$-semisimple, smooth representation of the Weil-Deligne group of $F$. We form the Langlands-Deligne local constant $\varepsilon\left(\sigma, s, \psi_{F}\right)$ of $\sigma$, relative to a complex variable $s$ and the character $\psi_{F}$.

Let $K / F$ be a finite, separable field extension and set $\psi_{K}=\psi_{F} \circ \operatorname{Tr}_{K / F}$. Let

$$
R_{K / F}=\operatorname{Ind}_{K / F} 1_{K}, \quad \delta_{K / F}=\operatorname{det} R_{K / F},
$$

where $1_{K}$ denotes the trivial representation of $\mathcal{W}_{K}$. We view $\delta_{K / F}$ as a character of $F^{\times}$. We define

$$
\lambda_{K / F}=\lambda_{K / F}\left(\psi_{F}\right)=\frac{\varepsilon\left(R_{K / F}, \frac{1}{2}, \psi_{F}\right)}{\varepsilon\left(1_{K}, \frac{1}{2}, \psi_{K}\right)} .
$$

If $F \subset K \subset E$, we have the relation

$$
\lambda_{E / F}=\lambda_{E / K} \lambda_{K / F}^{[E: K]}
$$

where $\lambda_{E / K}=\lambda_{E / K}\left(\psi_{K}\right)$.

For tamely ramified extensions $K / F$, we have the following table of values (see, for example, $[\mathrm{BF} 83, \S 10]$ or $[$ Tat79, $\S 3.2])$.

LEMma 1.5. Let $E / F$ be tamely ramified of degree $n$.

(1) If $E / F$ is unramified, then $\lambda_{E / F}=(-1)^{n-1}$.

(2) Suppose that $n$ is odd and that $E / F$ is totally ramified. The character $\delta_{E / F}$ is then unramified and

$$
\delta_{E / F}(\varpi)=\left(\frac{q}{n}\right)=\lambda_{E / F},
$$

for every prime element $\varpi$ of $F$.

(3) Suppose that $n=2$ and that $E / F$ is totally ramified; then

$$
\lambda_{E / F}=\mathfrak{w}\left(\psi_{F}\right) .
$$

\section{Main theorems}

Let $(E / F, \xi) \in P_{n}(F)$. Following the procedures of [BH05a, § 2] (see also the 'Background' section above), we get the irreducible supercuspidal representation ${ }_{F} \pi_{\xi} \in \mathcal{A}_{n}^{\text {et }}(F)$. On the other hand, we can form the irreducible representation ${ }_{F} \sigma_{\xi}=\operatorname{Ind}_{E / F} \xi$ of $\mathcal{W}_{F}$, and then the representation $\mathcal{L}\left({ }_{F} \sigma_{\xi}\right) \in \mathcal{A}_{n}^{\text {et }}(F)$, where $\mathcal{L}$ is the Langlands correspondence.

2.1 We know from [BH05a] (see Theorem $\mathrm{A}$ in the 'Introduction' above) that $\mathcal{L}\left({ }_{F} \sigma_{\xi}\right)$ is of the form ${ }_{F} \pi_{\mu \xi}$, for a character $\mu={ }_{F} \mu_{\xi}$ of $E^{\times}$such that $(E / F, \mu \xi) \in P_{n}(F)$ and

$$
\mu\left|U_{E}^{1}=1, \quad \mu\right| F^{\times}=\delta_{E / F} .
$$

Our main result identifies the character $F \mu_{\xi}$ when $E / F$ is totally ramified. We use the notation introduced in $\S 1$.

Theorem 2.1. Let $(E / F, \xi) \in P_{n}(F)$, and suppose that $E / F$ is totally ramified.

(1) Suppose that $n$ is odd; then

$$
{ }_{F} \mu_{\xi}(\varpi)=\left(\frac{q}{n}\right)=\lambda_{E / F}
$$

for every prime element $\varpi$ of $E$. 


\section{TAME LANGLANDS CORRESPONDENCE: TOTALLY RAMIFIED REPRESENTATIONS}

(2) Suppose that $n$ is even; then

$$
{ }_{F} \mu_{\xi}(\varpi)=\left(\frac{\zeta(\varpi, \xi)}{q}\right)\left(\frac{-1}{q}\right)^{\left(\boldsymbol{i}^{+}-1\right) / 2}\left(\frac{\boldsymbol{d}^{+}}{q}\right) \lambda_{E / F},
$$

for every prime element $\varpi$ of $E$.

We first observe that $F \mu_{\xi}$ is determined uniquely by (2.1.1) plus the identity of the theorem. Indeed, given (2.1.1), the character ${ }_{F} \mu_{\xi}$ is determined completely by the identities of the theorem at a single prime element $\varpi$.

Part (1) of the theorem follows from Lemma 1.5, part (2) and [BH05a, § 4.4]. We therefore concentrate on the case where $n$ is even.

Remark 2.1.1. The bijection $\mathcal{G}_{n}^{\text {et }}(F) \rightarrow \mathcal{A}_{n}^{\text {et }}(F)$, induced by $(E / F, \xi) \mapsto \operatorname{Ind}_{E / F} \xi$ and $(E / F, \xi) \mapsto$ ${ }_{F} \pi_{\xi},(E / F, \xi) \in P_{n}(F)$, is algebraic, in the sense that it respects the natural (external) action of Aut $\mathbb{C}$ on representations, while the Langlands correspondence is not [BH05a, Remarks 3.1 and 3.3]. It is in the presence of the irrationality $\lambda_{E / F}$ in Theorem 2.1, part (2) that this phenomenon first manifests itself.

Remark 2.1.2. Elaborating our notation to indicate the dependence on $\xi$, the parameters $\zeta(\varpi, \xi)$, $\boldsymbol{d}^{+}(\xi)$ and $\boldsymbol{i}^{+}(\xi)$ appearing in the expression for $\mu$ are essentially independent and random, in the following sense. We fix the extension $E / F$, totally tamely ramified of even degree $n$, and a prime element $\varpi$ of $E$. We choose a root of unity $\zeta \in \boldsymbol{\mu}_{F}$, a positive divisor $\boldsymbol{d}^{+}$of $n / 2$, and an odd positive integer $\boldsymbol{i}^{+}$. There is then a pair $(E / F, \xi) \in P_{n}(F)$ such that $\zeta(\varpi, \xi)=\zeta, \boldsymbol{d}^{+}(\xi)=\boldsymbol{d}^{+}$and $i^{+}(\xi)=i^{+}$.

Remark 2.1.3. The character ${ }_{F} \mu_{\xi}$ is necessarily independent of the choice of $\psi_{F}$. In the expression for $F \mu_{\xi}$ in the even-degree case, however, the first and last factors do vary with $\psi_{F}$.

\subsection{Example}

We choose odd prime numbers $p \neq l$; we put $F=\mathbb{Q}_{p}$ and we let $E / F$ be totally ramified of degree $2 l$. We next choose integers $k$ and $m$ with $0<k<m$; the integer $k$ is to be odd, while $m$ is to be even but not divisible by $l$. There is then an admissible pair $(E / F, \xi) \in P_{2 l}(F)$ for which $k$ and $m$ are the jumps of $\xi$. The field $K=E_{k}$ is of degree $l$ over $F$; the pair $(E / K, \xi)$ is admissible and $k$ is its only jump. We consider the character $F \mu_{\xi}$. According to Theorem 2.1, we have

$$
F \mu_{\xi}(\varpi)=\left(\frac{\zeta(\varpi)}{p}\right)\left(\frac{-1}{p}\right)^{(k-1) / 2}\left(\frac{l}{p}\right) \lambda_{E / F},
$$

for every prime element $\varpi$ of $E$.

The recipe in [Rei91] gives a conjectured value for $F \mu_{\xi}$ equal to what we would call $K \mu_{\xi}$. This character satisfies

$$
K \mu_{\xi}(\varpi)=\left(\frac{\zeta(\varpi)}{p}\right)\left(\frac{-1}{p}\right)^{(k-1) / 2} \lambda_{E / K} .
$$

We have $\lambda_{E / K}=\lambda_{E / F}$ from (1.5.2), so

$$
K \mu_{\xi}(\varpi)=\left(\frac{l}{p}\right){ }_{F} \mu_{\xi}(\varpi) .
$$

We can surely choose $l$ and $p$ to achieve ${ }_{K} \mu_{\xi} \neq{ }_{F} \mu_{\xi}$.

This example is, to a degree, typical: the approach of [Rei91] (incorporating [Moy86] and analogous work on representations of division algebras [BF83]) does not take sufficient account of the effect of the larger jumps. This is intrinsic to the method. 


\section{J. Bushnell and G. Henniart}

To give a brief summary, the approach of [Rei91] is based on a conjecture (or 'working hypothesis') equivalent to Theorem A. For $(E / F, \xi) \in P_{n}(F)$, the construction of $\pi={ }_{F} \pi_{\xi}$ in [Rei91] is equivalent to the one we have given in [BH05a]. The next step involves comparing the GodementJacquet local constants of $\xi$ and $\pi$. This gives partial information on ${ }_{F} \mu_{\xi}$. When the pair $(E / F, \xi)$ is minimal, in the sense that it has only one jump, this process determines ${ }_{F} \mu_{\xi}$ completely and correctly. In particular, the tables of [Rei91] give ${ }_{F} \mu_{\xi}$ correctly when $n$ is prime.

In the general situation, one can interpret the arguments of $\S 1$ above as showing that an admissible pair $(E / F, \xi)$ is 'glued' from a collection of minimal pairs $\left(E_{i-1} / E_{i}, \xi_{i}\right)$. The characters $\mu_{i}={ }_{E_{i}} \pi_{\xi_{i}}$ are known. The value for $F_{F} \mu_{\xi}$, as conjectured in [Rei91], is then obtained by glueing the $\mu_{i}$ in a process analogous to that for the pairs. The local constant comparisons provide only an approximate guide: [Rei91] chooses the simplest solution to all ambiguities.

For totally ramified pairs, the discrepancy between $F_{F} \mu_{\xi}$ and the version conjectured in [Rei91] is just as in the example above: it is unramified of order at most 2. As we shall see in the next paper of the series, the discrepancy can be ramified for more general admissible pairs.

2.3 Before starting the proof of Theorem 2.1, we derive a consequence of it. If $F^{\prime} / F$ is a finite cyclic extension, we denote by $\mathrm{b}_{F^{\prime} / F}$ the operation of base change, on irreducible smooth representations of groups $\mathrm{GL}_{n}(F)$, in the sense of [AC89] and [HL05].

We describe how the correspondence $(E / F, \xi) \mapsto{ }_{F} \pi_{\xi}$ behaves under unramified base change, in the case where $E / F$ is totally ramified.

Corollary 2.3. Let $(E / F, \xi) \in P_{n}(F)$, and suppose that $E / F$ is totally ramified. Let $L / F$ be unramified of degree $d$ and write $\xi_{L}=\xi \circ \mathrm{N}_{L E / E}$. Let $\nu$ denote the unramified character of $L E^{\times}$ such that

$$
\nu(\varpi)=(-1)^{(n-1)(d-1)},
$$

for every prime element $\varpi$ of $L E$. The pair $\left(L E / L, \nu \xi_{L}\right)$ is then admissible and

$$
\mathrm{b}_{L / F}\left({ }_{F} \pi_{\xi}\right)={ }_{L} \pi_{\nu \xi_{L}} .
$$

Proof. Write $\sigma=\operatorname{Ind}_{E / F} \xi \in \mathcal{G}_{n}^{\text {et }}(F)$; then $\operatorname{Ind}_{E L / L} \xi_{L}=\sigma_{L}=\sigma \mid \mathcal{W}_{L} \in \mathcal{G}_{n}^{\text {et }}(L)$. If we put $\pi=\mathcal{L}(\sigma)$ and $\pi_{L}=\mathcal{L}\left(\sigma_{L}\right)$, where $\mathcal{L}$ denotes the Langlands correspondence, we have $\pi_{L}=\mathrm{b}_{L / F} \pi$. Abbreviating $\mu={ }_{F} \mu_{\xi}$ and $\mu_{L}={ }_{L} \mu_{\xi_{L}}$, we therefore have to show that

$$
\mu_{L}=\nu \otimes \mu \circ \mathrm{N}_{E L / E} .
$$

We have $\delta_{E L / L}=\delta_{E / F} \circ \mathrm{N}_{L / F}$ so the relation (2.3.1) certainly holds in the case where $n$ is odd. In general, it is valid on $L^{\times} U_{E L}^{1}$. We may therefore assume $n$ is even, and we need only verify (2.3.1) at a single prime element $\varpi$ of $E L$. Indeed, we may take $\varpi$ to be a prime element of $E$.

We have to consider the invariants of the pair $\left(E L / L, \xi_{L}\right)$. One sees straight away that, in the notation of $\S 1$, we have $(E L)_{i}=E_{i} L$ for all integers $i \geqslant 0$. Thus the pairs $(E / F, \xi)$ and $\left(E L / L, \xi_{L}\right)$ have the same invariants $\boldsymbol{i}^{+}$and $\boldsymbol{d}^{+}$, while

$$
\zeta(\varpi, \xi)=\zeta\left(\varpi, \xi_{L}\right),
$$

when $\varpi$ is a prime element of $E$. Therefore, by Theorem 2.1,

$$
\mu_{L}(\varpi)=\left(\frac{\zeta(\varpi)}{q^{d}}\right)\left(\frac{-1}{q^{d}}\right)^{\left(\boldsymbol{i}^{+}-1\right) / 2}\left(\frac{\boldsymbol{d}^{+}}{q^{d}}\right) \lambda_{E L / L} .
$$

On the other hand, $\mathrm{N}_{E L / E}(\varpi)=\varpi^{d}$, so

$$
\mu\left(\mathrm{N}_{E L / E}(\varpi)\right)=\left(\frac{\zeta(\varpi)}{q^{d}}\right)\left(\frac{-1}{q^{d}}\right)^{\left(\boldsymbol{i}^{+}-1\right) / 2}\left(\frac{\boldsymbol{d}^{+}}{q^{d}}\right) \lambda_{E / F}^{d} .
$$


TAME LANGLANDS CORRESPONDENCE: TOTALLY RAMIFIED REPRESENTATIONS

We use (1.5.2) and Lemma 1.5 to get

$$
\lambda_{E L / L}=\lambda_{E L / L} \lambda_{L / F}^{n}=\lambda_{E L / F}=\lambda_{E L / E} \lambda_{E / F}^{d}=(-1)^{d-1} \lambda_{E / F}^{d},
$$

whence the result follows

It is interesting to note how the simplicity of this result fails to hint at the complexity of Theorem 2.1.

\section{Quadratic automorphic induction}

In this section, we take $(E / F, \xi) \in P_{n}(F)$, with $E / F$ totally ramified and $n=2 m$ even. We let $K / F$ be the unique quadratic sub-extension of $E / F$. Thus $(E / K, \xi) \in P_{m}(K)$, and we may form the representation $\rho={ }_{K} \pi_{\xi} \in \mathcal{A}_{m}^{\text {et }}(K)$. We state results comparing ${ }_{F} \pi_{\xi}$ with the representation $\mathrm{A}_{K / F} \rho \in \mathcal{A}_{n}^{\text {et }}(F)$ which is automorphically induced by $\rho$. We then deduce Theorem 2.1 .

3.1 Here, we treat the case where $n=2 m$, with $m$ odd.

Theorem 3.1. Suppose that $n=2 m$, with $m$ odd. Let $K / F$ be the quadratic subfield of $E / F$. Set $\rho={ }_{K} \pi_{\xi}$ and let $\pi=\mathrm{A}_{K / F} \rho$. There is then a tamely ramified character $\mu={ }_{K / F} \mu_{\xi}$ of $E^{\times}$, such that $\pi={ }_{F} \pi_{\mu \xi}$. The character ${ }_{K / F} \mu_{\xi}$ satisfies

$$
K / F \mu_{\xi} \mid F^{\times}=\delta_{K / F}^{m}
$$

and, for every prime element $\varpi$ of $E$ :

$$
{ }_{K / F} \mu_{\xi}(\varpi)=\left(\frac{-1}{q}\right)^{\left(i^{+}-1\right) / 2+\left(\boldsymbol{d}^{+}-1\right) / 2}\left(\frac{\boldsymbol{d}^{+}}{q}\right)\left(\frac{\zeta(\varpi)}{q}\right) \mathfrak{w}\left(\psi_{F}\right)^{m / \boldsymbol{d}^{+}} .
$$

These conditions determine it uniquely.

The existence of the character $\mu={ }_{K / F} \mu_{\xi}$, satisfying (3.1.1), is given by [BH05a, § 3.4]. Since $\mu \mid$ $F^{\times} U_{E}^{1}$ is already prescribed, the condition (3.1.2) determines $\mu$ completely. Indeed, $\mu$ is determined uniquely once (3.1.2) holds for one prime element $\varpi$.

In the present case, the character $\delta_{K / F}$ is ramified and of order 2. Thus $\mu \mid U_{F}$ has order 2, and the unramified character $\mu^{2}$ satisfies $\mu^{2}(\varpi)=\left(\frac{-1}{q}\right)$, for any prime element $\varpi$ of $E$.

3.2 We now consider the case where $n=2 m$, with $m$ even.

Theorem 3.2. Suppose that $n=2 m$, with $m$ even. Let $K / F$ be the quadratic subfield of $E / F$, write $\rho={ }_{K} \pi_{\xi}$ and $\pi=\mathrm{A}_{K / F} \rho$. There is then a tamely ramified character $\mu={ }_{K / F} \mu_{\xi}$ of $E^{\times}$such that $\pi={ }_{F} \pi_{\mu \xi}$. The character $\mu$ satisfies $\mu \mid F^{\times}=\delta_{K / F}^{m}=1$ and

$$
K / F \mu_{\xi}(\varpi)= \begin{cases}\left(\frac{-1}{q}\right)^{n / 4}\left(\frac{2}{q}\right) & \text { if } \boldsymbol{i}_{+} \text {is even, } \\ \left(\frac{-1}{q}\right)^{n / 4} & \text { if } \boldsymbol{i}_{+} \text {is odd }\end{cases}
$$

for every prime element $\varpi$ of $E$.

In this case, we note that $\mu$ is unramified and $\mu^{2}=1$. Again, $\mu$ is uniquely determined by (3.2.1). 


\section{J. Bushnell and G. Henniart}

3.3 We can now give the critical inductive step.

Corollary 3.3. Let $n$ be even, let $(E / F, \xi) \in P_{n}(F)$, and suppose that $E / F$ is totally ramified. Let $K / F$ be the unique quadratic sub-extension of $E / F$. We then have

$$
{ }_{F} \mu_{\xi}={ }_{K / F} \mu_{\xi} \cdot K \mu_{\xi}
$$

Proof. We put $\sigma_{K}=\operatorname{Ind}_{E / K} \xi$ and $\sigma=\operatorname{Ind}_{E / F} \xi=\operatorname{Ind}_{K / F} \sigma_{K}$. We then have

$$
{ }_{K} \mathcal{L}\left(\sigma_{K}\right)={ }_{K} \pi_{\xi^{\prime}}, \quad{ }_{F} \mathcal{L}(\sigma)={ }_{F} \pi_{\xi^{\prime \prime}},
$$

where $\xi^{\prime}={ }_{K} \mu_{\xi} \cdot \xi$ and $\xi^{\prime \prime}={ }_{F} \mu_{\xi} \cdot \xi$. However,

$$
{ }_{F} \mathcal{L}(\sigma)=\mathrm{A}_{K / F}\left({ }_{K} \mathcal{L}\left(\sigma_{K}\right)\right)={ }_{F} \pi_{\mu^{\prime} \xi^{\prime}}
$$

where $\mu^{\prime}={ }_{K / F} \mu_{\xi^{\prime}}$ (cf. [BH05a, $\S 3.2$, Proposition 6]). Theorems 3.1 and 3.2 show that the character $K / F \mu_{\xi^{\prime}}$ depends only on $\xi^{\prime} \mid U_{E}^{1}$, so $\mu^{\prime}={ }_{K / F} \mu_{\xi}$. We deduce that the admissible pairs $\left(E / F,{ }_{F} \mu_{\xi}\right)$ and $\left(E / F,{ }_{K / F} \mu_{\xi} \cdot K \mu_{\xi} \cdot \xi\right)$ are isomorphic, that is, the characters ${ }_{F} \mu_{\xi}$ and ${ }_{K / F} \mu_{\xi} \cdot K \mu_{\xi} \cdot \xi$ are $F$-conjugate. This conjugation fixes $\xi \mid U_{E}^{1}$ and, since $(E / F, \xi)$ is admissible, it must be trivial. The result follows.

3.4 The proofs of Theorems 3.1 and 3.2 occupy the remainder of the paper, starting in the next section. Here, we use them to prove Theorem 2.1, part (2).

We first treat the case where $m=n / 2$ is odd. Let $K / F$ be the quadratic sub-extension of $E / F$. By Corollary 3.3, we have ${ }_{F} \mu_{\xi}={ }_{K / F} \mu_{\xi} \cdot K \mu_{\xi}$. If $\varpi$ is a prime element of $E$, then

$$
K \mu_{\xi}(\varpi)=\left(\frac{q}{m}\right) .
$$

On the other hand,

$$
K / F \mu_{\xi}(\varpi)=\left(\frac{\zeta(\varpi)}{q}\right)\left(\frac{-1}{q}\right)^{\left(\boldsymbol{i}^{+}-1\right) / 2+\left(\boldsymbol{d}^{+}-1\right) / 2}\left(\frac{\boldsymbol{d}^{+}}{q}\right) \mathfrak{w}\left(\psi_{F}\right)^{m / \boldsymbol{d}^{+}} .
$$

We use the reciprocity relation

$$
\left(\frac{\boldsymbol{d}^{+}}{q}\right)=\left(\frac{-1}{q}\right)^{\left(\boldsymbol{d}^{+}-1\right) / 2}\left(\frac{q}{\boldsymbol{d}^{+}}\right)
$$

to get

$$
F \mu_{\xi}(\varpi)=\left(\frac{q}{m / \boldsymbol{d}^{+}}\right)\left(\frac{-1}{q}\right)^{\left(\boldsymbol{i}^{+}-1\right) / 2}\left(\frac{\zeta(\varpi)}{q}\right) \mathfrak{w}\left(\psi_{F}\right)^{m / \boldsymbol{d}^{+}} .
$$

We temporarily write $L=E_{\boldsymbol{i}^{+}}$and use the relation $\lambda_{E / F}=\lambda_{E / L K} \lambda_{L K / L}^{m / \boldsymbol{d}^{+}} \lambda_{L / F}^{n / \boldsymbol{d}^{+}}$. The first factor is

$$
\lambda_{E / L K}=\left(\frac{q}{m / \boldsymbol{d}^{+}}\right),
$$

while $\lambda_{L K / L}=\mathfrak{w}\left(\psi_{L}\right)$. As character of $\mathbb{k}_{L}=\mathbb{k}_{F}$, we have $\psi_{L}=\psi_{F} \circ \boldsymbol{d}^{+}$, so

$$
\mathfrak{w}\left(\psi_{F}\right)=\left(\frac{\boldsymbol{d}^{+}}{q}\right) \lambda_{L K / L} .
$$

Finally, $\lambda_{L / F}= \pm 1$ and $n / \boldsymbol{d}^{+}$is even so, altogether, we get

$$
F \mu_{\xi}(\varpi)=\left(\frac{-1}{q}\right)^{\left(\boldsymbol{i}^{+}-1\right) / 2}\left(\frac{\boldsymbol{d}^{+}}{q}\right)\left(\frac{\zeta(\varpi)}{q}\right) \lambda_{E / F},
$$

as required. 


\section{TAME LANGLANDS CORRESPONDENCE: TOTALLY RAMIFIED REPRESENTATIONS}

We now proceed inductively. We assume $n \equiv 0(\bmod 4)$ and let $K / F$ be the quadratic subextension of $E / F$. The extensions $E / K$ and $E / F$ have the same invariants $\boldsymbol{i}^{+}$and $\zeta(\varpi)$, while (in the obvious notation) $\boldsymbol{d}^{+}(E / F)$ could be $\boldsymbol{d}^{+}(E / K)$ or $2 \boldsymbol{d}^{+}(E / K)$. Inductively,

$$
K \mu_{\xi}(\varpi)=\left(\frac{-1}{q}\right)^{\left(\boldsymbol{i}^{+}-1\right) / 2}\left(\frac{\boldsymbol{d}^{+}(E / K)}{q}\right)\left(\frac{\zeta(\varpi)}{q}\right) \lambda_{E / K} .
$$

If $\boldsymbol{d}^{+}(E / F)=\boldsymbol{d}^{+}(E / K)$, the invariant $\boldsymbol{i}_{+}$of $E / F$ is odd and we are in the second case of $\S 3.2$. We have

$$
\lambda_{K / F}^{n / 2}=\left(\frac{-1}{q}\right)^{n / 4},
$$

and the result follows. We work similarly in the other case, where $\boldsymbol{d}^{+}(E / F)=2 \boldsymbol{d}^{+}(E / K)$ and $\boldsymbol{i}_{+}$ is even.

3.5 As the first step towards the proofs of Theorems 3.1 and 3.2, we interpret the constructions of $\S 1$ in terms of simple strata and characters.

Let $[\mathfrak{A}, l, 0, \beta]$ be a simple stratum in $A=\mathrm{M}_{n}(F)$. For an integer $i, 0 \leqslant i \leqslant l-1$, we may choose a simple stratum $[\mathfrak{A}, l, i, \gamma]$ equivalent to $[\mathfrak{A}, l, i, \beta]$ (see $[\mathrm{BK} 93, \S 2.4 .2])$. The integer $d_{i}=d_{i}(\beta, \mathfrak{A})=$ $[F[\gamma]: F]$ is then independent of choices. We set $d_{i}=1$, for $i \geqslant l$. We thus have $1 \leqslant d_{i} \leqslant[F[\beta]: F]$, and $d_{i}$ divides $d_{i-1}, i \geqslant 1$ (again see [BK93, $\left.\S 2.4 .2\right]$ ).

We say that $i$ is a jump of $\beta$ (relative to $\mathfrak{A}$ ) if $d_{i} \neq d_{i-1}$. We denote by $S(\beta, \mathfrak{A})$ this set of jumps.

3.6 Let $(E / F, \xi) \in P_{n}(F)$ and assume, for simplicity, that $E / F$ is totally ramified. As in [BH05a, $\S 1]$, there is a simple stratum $[\mathfrak{A}, l, 0, \beta]$ in $A$ and a simple character $\theta \in \mathcal{C}\left(\mathfrak{A}, \beta, \psi_{F}\right)$ with the following properties. First, the field $F[\beta]$ is $F$-isomorphic to $E$. Identifying $E$ with $F[\beta]$ (and so embedding $E$ in $A$ ), we have $H^{1}(\beta, \mathfrak{A}) \cap E^{\times}=U_{E}^{1}$, and $\theta \mid U_{E}^{1}=\xi$. The conjugacy class in $G=\mathrm{GL}_{n}(F)$ of the simple character $\theta$ with these properties is uniquely determined by the $F$-isomorphism class of $(E / F, \xi)$ (see [BH05a, $\S 1]$ ).

Proposition 3.6. Let $(E / F, \xi) \in P_{n}(F)$ and suppose that $E / F$ is totally ramified. Let $\theta \in$ $\mathcal{C}\left(\mathfrak{A}, \beta, \psi_{F}\right)$ be associated to $(E / F, \xi)$ as above.

(1) We have $S(\xi)=S(\beta, \mathfrak{A})$, for all choices of $[\mathfrak{A}, l, 0, \beta]$.

(2) One may choose $[\mathfrak{A}, l, 0, \beta]$ with the following additional properties (after identifying $F[\beta]$ with $E)$ :

(a) for each $i \in S(\xi)$, there is a simple stratum $\left[\mathfrak{A}, l, i, \gamma_{i}\right]$ in $A$, equivalent to $[\mathfrak{A}, l, i, \beta]$, such that $F\left[\gamma_{i}\right]=E_{i}$;

(b) for each $i \in S(\xi)$, we have $\beta-\gamma_{i} \equiv \alpha_{i}\left(\bmod \mathfrak{p}_{E}^{1-i}\right)$, where $\alpha_{i}$ is defined in $\S 1.1$.

Proof. We first remark that if we have simple strata $\left[\mathfrak{A}, l_{i}, 0, \beta_{i}\right]$ in $A$, and characters $\theta_{i} \in \mathcal{C}\left(\mathfrak{A}, \beta_{i}, \psi_{F}\right)$ which intertwine in $G$, for $i=1,2$, then $S\left(\beta_{1}, \mathfrak{A}\right)=S\left(\beta_{2}, \mathfrak{A}\right)$, as follows from [BK93, (3.5.1) and (3.5.11)].

We view $E$ as embedded in $A$, and we let $\mathfrak{A}$ be the unique hereditary $\mathfrak{o}_{F}$-order in $A$ which is stable under conjugation by $E^{\times}$.

We proceed by induction on $|S(\xi)|$. Suppose first that $|S(\xi)|=1$. Replacing $\xi$ by $\xi \otimes \chi \circ \mathrm{N}_{E / F}$, for a suitable character $\chi$ of $F^{\times}$, we can assume that $S(\xi)=\{l\}$. We then have $\operatorname{gcd}(l, n)=1$. We choose an element $\beta \in E$ such that $\xi \mid U_{E}^{[l / 2]+1}=\psi_{E, \beta}$. Since $l$ is the only jump of $\xi$, we have $E=F[\beta]$, for any choice of $\beta$ here. Since $l=-v_{E}(\beta)$ is relatively prime to $n=e(E \mid F)$, the stratum $[\mathfrak{A}, l, l-1, \beta]$ is simple, whence so is $[\mathfrak{A}, l, 0, \beta]$. There exists $\theta \in \mathcal{C}\left(\mathfrak{A}, \beta, \psi_{F}\right)$ extending $\xi \mid U_{E}^{1}$. Since $[\mathfrak{A}, l, l-1, \beta]$ is simple, we have $S(\beta)=\{l\}$, as required. 


\section{J. Bushnell and G. Henniart}

We now assume that $|S(\xi)| \geqslant 2$, and we let $i$ be the least element of $S(\xi)$. Write $K=E_{i}$; we factorize $\xi$ as in $\S 1$ :

$$
\begin{aligned}
\xi \mid U_{E}^{1} & =\phi \otimes \chi \circ \mathrm{N}_{E / K}, \\
\phi \mid U_{E}^{[i / 2]+1} & =\psi_{E, \alpha},
\end{aligned}
$$

for a quasicharacter $\chi$ of $K^{\times}$and an element $\alpha \in E$ of valuation $-i$. For any such choices, we have $E=K[\alpha]$ and $-i=v_{E}(\alpha)$ is relatively prime to $e=e(E \mid K)=[E: K]$.

The pair $(K / F, \chi)$ is admissible and $S(\xi)=\{i\} \cup\{e j: j \in S(\chi)\}$. The pair $(K / F, \chi)$ gives a simple stratum $\left[\mathfrak{A}_{0}, l / e, 0, \gamma\right]$ in $A_{0}=\mathrm{M}_{n / e}(K)$ and a simple character $\tau_{0} \in \mathcal{C}\left(\mathfrak{A}_{0}, \gamma, \psi_{F}\right)$ such that $F[\gamma]=K$ and $\tau_{0} \mid U_{K}^{1}=\chi$. Inductively, $S\left(\gamma, \mathfrak{A}_{0}\right)=S(\chi)$ and property $(2)(\mathrm{b})$ holds for $\chi$.

The element $\gamma \in E$ gives a simple stratum $[\mathfrak{A}, l, 0, \gamma]$ in $A$ and there is a simple character $\tau \in \mathcal{C}\left(\mathfrak{A}, \gamma, \psi_{F}\right)$ which is endo-equivalent to $\tau_{0}$. If $B$ denotes the $A$-centralizer of $K$ and $\mathfrak{B}=B \cap \mathfrak{A}$, then $\tau \mid U_{\mathfrak{B}}^{1}=\chi \circ \operatorname{det}_{B}$.

The stratum $[\mathfrak{B}, i, i-1, \alpha]$ in $B$ is simple and $K[\alpha]=E$. There is a tame corestriction $s_{\gamma}: A \rightarrow B$, relative to $K / F$, which is the identity map on $B$ (see [BK93, (1.3.8)]). Set $\beta=\gamma+\alpha$. We deduce from $[\mathrm{BK} 93,(2.2 .3)]$ that $[\mathfrak{A}, l, 0, \beta]$ is simple with $E=F[\beta]$, and that

$$
\begin{aligned}
S(\beta, \mathfrak{A}) & =\{i\} \cup S(\gamma, \mathfrak{A})=\{i\} \cup\left\{e j: j \in S\left(\gamma, \mathfrak{A}_{0}\right)\right\} \\
& =\{i\} \cup\{e j: j \in S(\chi)\}=S(\xi) .
\end{aligned}
$$

We have $H^{1}(\beta, \mathfrak{A})=U_{E}^{1} H^{[i / 2]+1}(\gamma, \mathfrak{A})$; the character

$$
\theta: x h \longmapsto \phi(x) \tau(h), \quad x \in U_{E}^{1}, h \in H^{[i / 2]+1}(\gamma, \mathfrak{A}),
$$

lies in $\mathcal{C}\left(\mathfrak{A}, \beta, \psi_{F}\right)$ and $\theta \mid U_{E}^{1}=\xi$, as required.

\section{Representations in finite classical groups}

The proofs of Theorems 3.1 and 3.2 rest on calculations requiring a number of elementary properties of the representations, linear, orthogonal and symplectic, of a finite cyclic group over a finite field. We collect in this section the results and notation we shall need. The proofs are either straightforward exercises or parallel to arguments in [BF83]. We have therefore omitted them.

Throughout, $\Gamma$ denotes a cyclic group of order $n$ and $\mathbb{k}$ a finite field of $q$ elements and characteristic $p$. We always assume that $p \nmid n$ and, for much of the time, that $p \neq 2$.

4.1 Let $\overline{\mathbb{k}} / \mathbb{k}$ be an algebraic closure of $\mathbb{k}$, and write $\Omega=\operatorname{Gal}(\overline{\mathbb{k}} / \mathbb{k})$. Let $\widehat{\Gamma}=\operatorname{Hom}\left(\Gamma, \overline{\mathbb{k}}^{\times}\right)$. Thus $\Omega$ acts on $\widehat{\Gamma}$ : we denote this action by $\omega: \chi \mapsto \omega \chi, \omega \in \Omega, \chi \in \widehat{\Gamma}$.

To $\chi \in \widehat{\Gamma}$ we attach a $\mathbb{k} \Gamma$-module $V_{\chi}$ as follows: the underlying vector space $V_{\chi}$ is the field extension $\mathbb{k}[\chi] / \mathbb{k}$ generated by the values of $\chi$, and $\Gamma$ acts via the character $\chi: \Gamma \rightarrow \mathbb{k}[\chi]^{\times}$.

Lemma 4.1. The $\mathbb{k} \Gamma$-module $V_{\chi}$ is simple, and its isomorphism class depends only on the $\Omega$-orbit of $\chi$. The process $\chi \mapsto V_{\chi}$ induces a bijection between the set of $\Omega$-orbits in $\widehat{\Gamma}$ and the set of isomorphism classes of simple $\mathbb{k} \Gamma$-modules.

In particular, we have

$$
\mathbb{k} \Gamma=\bigoplus_{\chi \in \Omega \backslash \widehat{\Gamma}} V_{\chi}
$$

It will be useful to have some standard $\Gamma$-submodules of $\mathbb{k} \Gamma$. First, let $a$ be a positive divisor of $n$; thus $\Gamma^{a}$ is the subgroup of $\Gamma$ of order $n / a$, and we identify the group ring $\mathbb{k}\left[\Gamma / \Gamma^{a}\right]$ with the set 
of $\Gamma^{a}$-fixed points in $\mathbb{k} \Gamma$. There is a unique $\Gamma$-submodule $I_{\mathbb{k}}(\Gamma ; a, n)$ of $\mathbb{k} \Gamma$ such that

$$
\mathbb{k} \Gamma=\mathbb{k}\left[\Gamma / \Gamma^{a}\right] \oplus I_{\mathbb{k}}(\Gamma ; a, n) .
$$

Explicitly,

$$
\mathbb{k}\left[\Gamma / \Gamma^{a}\right]=\bigoplus_{\substack{\chi \in \Omega \backslash \widehat{\Gamma}, \chi^{a}=1}} V_{\chi}, \quad I_{\mathbb{k}}(\Gamma ; a, n)=\bigoplus_{\substack{\chi \in \Omega \backslash \widehat{\Gamma}, \chi^{a} \neq 1}} V_{\chi} .
$$

More generally, if $a|b| n$, we define $I_{\mathbb{k}}(\Gamma ; a, b)$ by

$$
\mathbb{k}\left[\Gamma / \Gamma^{b}\right]=\mathbb{k}\left[\Gamma / \Gamma^{a}\right] \oplus I_{\mathbb{k}}(\Gamma ; a, b) .
$$

4.2 We shall use these notions in the following context. We have a simple stratum $[\mathfrak{A}, l, 0, \beta]$ in $\mathrm{M}_{n}(F)$ with $E=F[\beta] / F$ totally tamely ramified of degree $n$ (so, in particular, $p \nmid n$ ). The order $\mathfrak{A}$ is then principal; we put $\mathfrak{P}=\operatorname{rad} \mathfrak{A}$. The quotient ring $\mathfrak{A} / \mathfrak{P}$ is isomorphic to $\mathbb{k}_{F} \times \cdots \times \mathbb{k}_{F}=\mathbb{k}_{F}^{n}$ as $\mathbb{k}_{F}$-algebra.

The group $\Gamma=E^{\times} / F^{\times} U_{E}^{1}$ is cyclic of order $n$; it acts, by conjugation, on $\mathfrak{A} / \mathfrak{P}$ by permuting simple components. Thus we have an isomorphism $\mathfrak{A} / \mathfrak{P} \cong \mathbb{k}_{F} \Gamma$ of $\Gamma$-modules, taking indecomposable idempotents of $\mathfrak{A} / \mathfrak{P}$ to elements of $\Gamma$. This isomorphism is determined up to translation by an element of $\Gamma$, and is adequately unique for our purposes.

It follows that, for a dividing $n, \mathfrak{A} / \mathfrak{P}$ has a unique $\Gamma$-submodule $I_{\mathfrak{A}}(a, n)$ isomorphic to $I_{\mathbb{k}_{F}}(\Gamma ; a, n)$. If $K / F$ is the sub-extension of $E / F$ such that $[E: K]=a$ and if $\mathfrak{A}_{K}$ is the $\mathfrak{A}$-centralizer of $K$, then $\mathfrak{A}_{K} / \mathfrak{P}_{K}$ is the set of $K^{\times}$-fixed points in $\mathfrak{A} / \mathfrak{P}$. We have

$$
\mathfrak{A} / \mathfrak{P}=\mathfrak{A}_{K} / \mathfrak{P}_{K} \oplus I_{\mathfrak{A}}(a, n) .
$$

More generally, we may set $I_{\mathfrak{A}}(a, b) \cong I_{\mathbb{k}_{F}}(\Gamma ; a, b)$.

4.3 We return to the general notation of $\S 4.1$. Let $U$ be some finite $\mathbb{k} \Gamma$-module; the linear dual $U^{*}=\operatorname{Hom}_{\mathbb{k}}(U, \mathbb{k})$ is then a $\mathbb{k} \Gamma$-module in the natural way, and $\left(V_{\chi}\right)^{*}=V_{\chi^{-1}}$. We use this to divide the simple $\mathbb{k} \Gamma$-modules into three classes.

DeFinition 4.3. Let $U=V_{\chi}$ be a simple $\mathbb{k} \Gamma$-module, for some $\chi \in \widehat{\Gamma}$. We say that:

(1) $U$ is non-self-dual ( $n s d$ ) if $U \neq U^{*}$ or, equivalently, if $\Omega \chi^{-1} \neq \Omega \chi$;

(2) $U$ is trivially self-dual (tsd) if $\chi=\chi^{-1}$ or, equivalently, $\chi^{2}=1$;

(3) $U$ is non-trivially self-dual (ntsd) if $U \cong U^{*}$ but $\chi^{2} \neq 1$.

In case (3), we have $\chi \neq \chi^{-1}$ but $\Omega \chi^{-1}=\Omega \chi$. This case arises as follows. First, the degree $[\mathbb{k}[\chi]: \mathbb{k}]=2 d$ is even. Let $\mathbb{k}_{1} / \mathbb{k}$ have degree $d$, and let $\mathcal{T}$ denote the kernel of the norm map $\mathrm{N}_{\mathbb{k}[\chi] / \mathbb{k}_{1}}$. We then have $\chi(\Gamma) \subset \mathcal{T}$.

4.4 Until further notice, we assume $p \neq 2$. Let $(V, h)$ be an orthogonal representation of $\Gamma$ over $\mathbb{k}$. Thus $V$ is a finite $\mathbb{k} \Gamma$-module and $h: V \times V \rightarrow \mathbb{k}$ is a non-degenerate, symmetric bilinear form satisfying

$$
h\left(\gamma v_{1}, \gamma v_{2}\right)=h\left(v_{1}, v_{2}\right), \quad v_{i} \in V, \gamma \in \Gamma .
$$

Any such representation can be written as an orthogonal sum

$$
(V, h)=\left(V_{1}, h_{1}\right) \perp\left(V_{2}, h_{2}\right) \perp \cdots \perp\left(V_{r}, h_{r}\right),
$$

in which the factors $\left(V_{j}, h_{j}\right)$ are indecomposable orthogonal representations of $\Gamma$. Such a decomposition is unique up to $\Gamma$-isometry and permutation of the factors. It is a simple matter to classify the indecomposable orthogonal representations, as follows. 


\section{J. Bushnell and G. Henniart}

Lemma 4.4. Let $(V, h)$ be an indecomposable orthogonal representation of $\Gamma$ over $\mathbb{k}$. Exactly one of the following holds:

(1) $V$ is a simple $\mathbb{k} \Gamma$-module of tsd type;

(2) $(V, h)$ is isometric to $\left(U \oplus U^{*}, h_{U}\right)$, where $U$ is a simple $\mathbb{k} \Gamma$-module of nsd type and

$$
h_{U}\left(\left(u_{1}, u_{1}^{*}\right),\left(u_{2}, u_{2}^{*}\right)\right)=\left\langle u_{1}, u_{2}^{*}\right\rangle+\left\langle u_{2}, u_{1}^{*}\right\rangle, \quad u_{i} \in U, u_{i}^{*} \in U^{*},
$$

where $\langle$,$\rangle is the canonical pairing U \times U^{*} \rightarrow \mathbb{k}$;

(3) $V=V_{\chi}$ is ntsd and the form $h$ is given, up to $\Gamma$-isometry, by

$$
h:(x, y) \longmapsto \operatorname{Tr}_{\mathbb{k}[\chi] / \mathbb{k}}(x \bar{y}),
$$

where $x \mapsto \bar{x}$ is the non-trivial automorphism of $\mathbb{k}[\chi] / \mathbb{k}$ of order 2 .

If $V$ is a finite $\mathbb{k}$-vector space and $h: V \times V \rightarrow \mathbb{k}$ is a non-degenerate symmetric bilinear form, the isometry class of $h$ is determined by the quantity $\operatorname{det} h$ modulo $\mathbb{k}^{\times 2}$. When $(V, h)$ is also an orthogonal representation of $\Gamma$, one can compute the discriminant $\operatorname{det} h\left(\bmod \mathbb{k}^{\times 2}\right)$, as follows.

Proposition 4.4. Using the notation of Lemma 4.4, we have

$$
\left(\frac{\operatorname{det} h}{q}\right)= \begin{cases}\left(\frac{-1}{q}\right)^{\operatorname{dim} V / 2} & \text { in case (2), } \\ -\left(\frac{-1}{q}\right)^{\operatorname{dim} V / 2} & \text { in case (3). }\end{cases}
$$

In case (1) of the lemma, det $h$ is arbitrary.

In cases $(2)$ and $(3)$, therefore, the orthogonal $\mathbb{k} \Gamma$-module $(V, h)$ is uniquely determined by the underlying $\mathbb{k} \Gamma$-module $V$.

4.5 We take a finite $\mathbb{k}$-vector space $V$ and a quadratic form $Q: V \rightarrow \mathbb{k}$. Let $\psi$ be some non-trivial character of $\mathbb{k}$, and consider the Gauss sum

$$
\mathfrak{g}(Q, \psi)=\sum_{v \in V} \psi(Q(v)) .
$$

In particular, if $V=\mathbb{k}$ and $Q(x)=x^{2}$, we get

$$
\mathfrak{g}(Q, \psi)=\sum_{x \in \mathbb{k}} \psi\left(x^{2}\right)=\sum_{y \in \mathbb{k}^{\times}}\left(\frac{y}{q}\right) \psi(y) .
$$

In this case, we write

$$
\mathfrak{g}(Q, \psi)=\mathfrak{g}(\psi)
$$

In general, we may write $Q(v)=h(v, v)$, where $h$ is the symmetric bilinear form

$$
h\left(v_{1}, v_{2}\right)=\frac{1}{2}\left(Q\left(v_{1}+v_{2}\right)-Q\left(v_{1}\right)-Q\left(v_{2}\right)\right) .
$$

Thus $Q$ is non-degenerate if and only if $\operatorname{det} h \neq 0$; in this case, we put $\operatorname{det} Q=\operatorname{det} h$ (and think of this as an element of $\left.\mathbb{k}^{\times} / \mathbb{k}^{\times 2}\right)$. In general, we may write $Q$ as an orthogonal sum $Q=Q_{0} \perp Q_{\mathrm{nd}}$, where $Q_{0}$ is null and $Q_{\text {nd }}$ is non-degenerate. We obtain the following proposition.

Proposition 4.5. Let $|\mathbb{k}|=q$. We have

$$
\mathfrak{g}(Q, \psi)=\left(\frac{\operatorname{det} Q_{\mathrm{nd}}}{q}\right) \mathfrak{g}(\psi)^{\operatorname{dim} Q_{\mathrm{nd}}} q^{\operatorname{dim} Q_{0}}
$$




\section{TAME LANGLANDS CORRESPONDENCE: TOTALLY RAMIFIED REPRESENTATIONS}

Remark 4.5. Let $(V, h)$ be an orthogonal representation of $\mathbb{k} \Gamma$; the quadratic form $Q: v \mapsto h(v, v)$ is then (by definition) non-degenerate and $\Gamma$-invariant. Let $V_{2}$ denote the subspace of $V$ fixed by $\Gamma^{2}$. We have an orthogonal decomposition $V=V_{2} \perp V_{2}^{\prime}$; the discriminant $\operatorname{det} h$, and hence the Gauss sum $\mathfrak{g}(Q, \psi)$, is determined by the $\mathbb{k} \Gamma$-module $V$ and the restriction of $h$ to $V_{2}$ (cf. $\S 4.4$ ).

4.6 The group ring $\mathbb{k} \Gamma$ carries the standard $\Gamma$-invariant quadratic form $Q_{\Gamma}$ for which the group elements $\gamma \in \Gamma$ form an orthonormal basis. Thus $Q_{\Gamma}$ is non-degenerate and $\operatorname{det} Q_{\Gamma}=1$.

Let $\varepsilon_{\Gamma}: \mathbb{k} \Gamma \rightarrow \mathbb{k}$ be the augmentation map, and let $x \mapsto \bar{x}$ be the $\mathbb{k}$-linear involution on $\mathbb{k} \Gamma$ such that $\bar{\gamma}=\gamma^{-1}, \gamma \in \Gamma$. We then have $Q_{\Gamma}(x)=\varepsilon_{\Gamma}(x \bar{x}), x \in \mathbb{k} \Gamma$.

In the context of $\S 4.2$, there are parallel structures on the ring $\mathfrak{A} / \mathfrak{P}$. The algebra $\operatorname{trace} \operatorname{tr}_{A}$ on $A=\mathrm{M}_{n}(F)$ reduces to the algebra trace $\mathfrak{A} / \mathfrak{P} \rightarrow \mathbb{k}_{F}$. Under the isomorphism $\mathfrak{A} / \mathfrak{P} \cong \mathbb{k}_{F} \Gamma$ of $\S 4.2$, the quadratic form $Q_{\Gamma}$ then corresponds to the form $x \mapsto \operatorname{tr}_{A}\left(x^{2}\right)$ on $\mathfrak{A} / \mathfrak{P}$.

Let $a$ be a positive divisor of $n$. Consider the restriction of $Q_{\Gamma}$ to $\mathbb{k}\left[\Gamma / \Gamma^{a}\right] \subset \mathbb{k} \Gamma$ :

$$
Q_{\Gamma} \mid \mathbb{k}\left[\Gamma / \Gamma^{a}\right]=a^{-1} n Q_{\Gamma / \Gamma^{a}}, \quad \operatorname{det}\left(Q_{\Gamma} \mid \mathbb{k}\left[\Gamma / \Gamma^{a}\right]\right)=\left(a^{-1} n\right)^{a} .
$$

Therefore

$$
\operatorname{det}\left(Q_{\Gamma} \mid I_{\mathbb{k}}(\Gamma ; a, n)\right)=\left(a^{-1} n\right)^{a} .
$$

4.7 We may now allow the possibility $p=2$, but the base field $\mathbb{k}$ is replaced by the field $\mathbb{F}_{p}$ of $p$ elements.

Let $(V, h)$ be a symplectic representation of $\Gamma$ over $\mathbb{F}_{p}$; thus $V$ is a finite $\mathbb{F}_{p} \Gamma$-module and $h: V \times V \rightarrow \mathbb{F}_{p}$ is a non-degenerate, alternating, $\Gamma$-invariant bilinear form. Again, any symplectic representation of $\Gamma$ can be written as an orthogonal sum of indecomposable symplectic representations.

If $U$ is a finite $\mathbb{F}_{p} \Gamma$-module, we can define the hyperbolic space $H(U)=(V, h)$, where $V=U \oplus U^{*}$ and

$$
h\left(\left(u_{1}, u_{1}^{*}\right),\left(u_{2}, u_{2}^{*}\right)\right)=\left\langle u_{1}, u_{2}^{*}\right\rangle-\left\langle u_{2}, u_{1}^{*}\right\rangle, \quad u_{i} \in U, u_{i}^{*} \in U^{*} .
$$

With this notation, we have the following lemma.

Lemma 4.7. Let $(V, h)$ be an indecomposable symplectic representation of $\Gamma$ over $\mathbb{F}_{p}$. Exactly one of the following holds:

(1) $(V, h)=H(U)$, where $U$ is tsd or nsd;

(2) $V \cong V_{\chi}$ is ntsd and, up to $\Gamma$-isometry, $h$ is given by

$$
h:(x, y) \longmapsto \operatorname{Tr}_{\mathbb{F}_{p}[\chi] / \mathbb{F}_{p}}(x \alpha \bar{y}),
$$

where $x \mapsto \bar{x}$ is the automorphism of $\mathbb{F}_{p}[\chi] / \mathbb{F}_{p}$ of order 2 and $\alpha \in \mathbb{F}_{p}[\chi]^{\times}$satisfies $\bar{\alpha}=-\alpha$.

In all cases, the $\Gamma$-isometry class of $(V, h)$ is determined by the isomorphism class of the underlying $\mathbb{F}_{p} \Gamma$-module $V$.

In case (2), we recall, the character $\chi$ takes values in the group $\mathcal{T}$ of elements of the form $\bar{x} / x$, $x \in \mathbb{F}_{p}[\chi]^{\times}$.

Following [BF83], we define an invariant $t_{\Gamma}(V, h)$ of a symplectic representation $(V, h)$ of $\Gamma$ over $\mathbb{F}_{p}$. Since the isometry class of $(V, h)$ is determined by the isomorphism class of $V$, we usually write $t_{\Gamma}(V, h)=t_{\Gamma}(V)$.

This invariant satisfies

$$
t_{\Gamma}\left(\left(V_{1}, h_{1}\right) \perp\left(V_{2}, h_{2}\right)\right)=t_{\Gamma}\left(V_{1}, h_{1}\right) \perp t_{\Gamma}\left(V_{2}, h_{2}\right),
$$

so we need only define $t_{\Gamma}$ for indecomposable symplectic representations. 


\section{J. Bushnell and G. Henniart}

Definition 4.7. Let $(V, h)$ be an indecomposable symplectic $\mathbb{F}_{p} \Gamma$-module, and let $\gamma$ be a generator of $\Gamma$. Then we have the following.

(1) If $(V, h) \cong H\left(\mathbb{F}_{p}\right)$, where $\Gamma$ acts trivially on $\mathbb{F}_{p}$, then $t_{\Gamma}(V)=p$.

(2) Suppose $(V, h) \cong H(U)$, for some non-trivial, simple $\mathbb{F}_{p} \Gamma$-module $U$. Then $t_{\Gamma}(V)$ is the signature of the permutation of $U$ induced by the action of $\gamma$.

(3) Suppose that $(V, h)$ is anisotropic, $V \cong V_{\chi}$, say. Take $\mathcal{T} \subset \mathbb{F}_{p}[\chi]^{\times}$as above; then

$$
t_{\Gamma}(V)= \begin{cases}-1 & \text { if } \chi(\Gamma) \subset \mathcal{T}^{2} \\ +1 & \text { otherwise }\end{cases}
$$

Using only elementary arguments, one obtains the following properties of the invariant $t_{\Gamma}$.

Proposition 4.7. Let $(V, h)$ be a finite symplectic $\mathbb{F}_{p} \Gamma$-module.

(1) Suppose that $\Gamma$ has only the trivial fixed point in $V$ and that $V \cong H(U)$, for some $\Gamma$-module $U$. Then $t_{\Gamma}(V)$ is the signature of the permutation of $U$ induced by the action of $\gamma$, for any generator $\gamma$ of $\Gamma$.

(2) Let $\Delta$ be the 2-Sylow subgroup of $\Gamma$, and assume that $\Delta$ has only the trivial fixed point on $V$. Then $t_{\Gamma}(V)=t_{\Delta}(V)$.

4.8 The trace invariant $t_{\Gamma}$ appears in the following context. Let $\mathcal{G}$ be a finite, extra-special $p$-group of class 2 , with cyclic centre $\mathcal{Z}$, and write $\mathcal{V}=\mathcal{G} / \mathcal{Z}$. Let $\theta$ be a faithful character of $\mathcal{Z}$. The group $\mathcal{V}$ is an elementary abelian $p$-group, and the commutator pairing $h_{\theta}:(x, y) \mapsto \theta\left(x y x^{-1} y^{-1}\right)$ induces a non-degenerate alternating bilinear form on $\mathcal{V}$, with values in the group of $p$ th roots of unity. There is a unique irreducible representation $\eta$ of $\mathcal{G}$ such that $\eta \mid \mathcal{Z}$ contains $\theta$.

Let $\Gamma$ be a cyclic group of automorphisms of $\mathcal{G}$, of order $n$. We assume that $p \nmid n$, and that $\Gamma$ fixes $\mathcal{Z}$. It follows that $\Gamma$ fixes the pairing $h_{\theta}$, so $\left(\mathcal{V}, h_{\theta}\right)$ afford a symplectic representation of $\Gamma$ over $\mathbb{F}_{p}$.

Further, $\Gamma$ fixes $\eta$ and we may apply the Glauberman correspondence [Gla68]. Let $\tilde{\eta}$ be the unique representation of $\Gamma \ltimes \mathcal{G}$ such that $\tilde{\eta} \mid \mathcal{G} \cong \eta$ and $\operatorname{det} \tilde{\eta} \mid \Gamma=1$.

The group $\mathcal{G}^{\Gamma}$ has centre $\mathcal{Z}$ and, since $p \nmid n$, one sees easily that $\mathcal{G}^{\Gamma} / \mathcal{Z}=\mathcal{V}^{\Gamma}$. Moreover, $\mathcal{V}^{\Gamma}$ is a non-degenerate subspace of $\mathcal{V}$. Thus there is a unique irreducible representation $\zeta$ of $\mathcal{G}^{\Gamma}$ such that $\zeta \mid \mathcal{Z}$ contains $\theta$. It follows readily that $\zeta$ is the Glauberman correspondent of $\eta$, and there is a constant $\epsilon_{\Gamma}=\epsilon_{\Gamma}(\mathcal{G}, \eta)= \pm 1$ such that

$$
\operatorname{tr} \tilde{\eta}(\gamma x)=\epsilon_{\Gamma} \operatorname{tr} \zeta(x),
$$

for all $x \in \mathcal{G}^{\Gamma}$ and all generators $\gamma$ of $\Gamma$.

From [BF83, (8.6.1)] or [Wil89], we have the following proposition.

Proposition 4.8. The Glauberman sign $\epsilon_{\Gamma}$ is given by

$$
\epsilon_{\Gamma}=t_{\Gamma}(\mathcal{V}) /\left|t_{\Gamma}(\mathcal{V})\right|
$$

In the notation of the 'Background' section, we will apply Proposition 4.8 to the case $\mathcal{G}=\mathcal{J}=$ $J^{1} / \operatorname{Ker} \theta$, acted on by $\Gamma=E^{\times} / F^{\times} U_{E}^{1}$. The proposition then gives $\epsilon_{F}=t_{\Gamma}(\mathcal{J})$.

\section{Character values at special 1-units}

In this section, we take an admissible pair $(E / F, \xi) \in P_{n}(F)$ in which $E / F$ is totally ramified. In particular, $p \nmid n$. We form the representation $\pi={ }_{F} \pi_{\xi}$ of $G=\mathrm{GL}_{n}(F)$ and derive an expression 


\section{TAME LANGLANDS CORRESPONDENCE: TOTALLY RAMIFIED REPRESENTATIONS}

for the value of the character $\operatorname{tr} \pi$ at elements of the form $1+\varpi$, where $\varpi$ is a prime element of $E$. In the next section, we will use this to get an expression for the quantity $c \boldsymbol{\delta}(\varpi)$ appearing in Theorem $\mathrm{C}$ of the 'Introduction'.

Ultimately, therefore, we shall only be interested in the case where $n$ is even and hence $p \neq 2$. There is no need to impose such a restriction at the beginning.

5.1 Let $A=\mathrm{M}_{n}(F)$. As in [BH05a] or the summary in the 'Background' section above, the pair $(E / F, \xi)$ gives rise to a simple stratum $[\mathfrak{A}, l, 0, \beta]$ in $A$, such that $E=F[\beta]$, and a simple character $\theta \in \mathcal{C}\left(\mathfrak{A}, \beta, \psi_{F}\right)$ which agrees with $\xi$ on $U_{E}^{1}$. We abbreviate $H^{1}=H^{1}(\beta, \mathfrak{A})$, and similarly for $J^{1}$ and $J^{0}$. Let $\eta$ denote the unique irreducible representation of $J^{1}$ which contains $\theta$. We set $\boldsymbol{J}=E^{\times} J^{0}=E^{\times} J^{1}$. There is a unique irreducible representation $\Lambda$ of $\boldsymbol{J}$ such that $\Lambda \mid J^{1} \cong \eta$ and $\pi=c-\operatorname{Ind}_{J}^{G} \Lambda$.

Let $\varpi$ be a prime element of $E$. The character value $\operatorname{tr} \pi(1+\varpi)$ is given by

$$
\operatorname{tr} \pi(1+\varpi)=\sum_{x \in G / J} \operatorname{tr} \Lambda\left(x^{-1}(1+\varpi) x\right)
$$

(see [BH96, Appendix]). To calculate it, we must first determine the set of $x \in G$ for which $x^{-1}(1+$ $\varpi) x \in \boldsymbol{J}$.

Lemma 5.1. Let $x \in G$ and suppose that $x^{-1}(1+\varpi) x \in \boldsymbol{J}$. Then

(1) $x \in \mathcal{K}_{\mathfrak{A}}$,

(2) $x^{-1} \varpi x \in \mathcal{K}_{\mathfrak{A}}$, and

(3) $x^{-1}(1+\varpi) x \in J^{1}$.

Proof. The element $x^{-1}(1+\varpi) x$ of $\boldsymbol{J}$ is pro-unipotent, so it lies in $J^{1}$. In particular, $x^{-1} \varpi x$ lies in $\mathfrak{P}=\operatorname{rad} \mathfrak{A}$. Since $v_{F}\left(\operatorname{det} x^{-1} \varpi x\right)=1$, this element must generate $\mathfrak{P}$ as $\mathfrak{A}$-module, and so lies in $\mathcal{K}_{\mathfrak{A}}$.

Since $\varpi$ generates a field and is minimal over $F$, the fact $x^{-1} \varpi x \in \mathcal{K}_{\mathfrak{A}}$ implies that $F\left[x^{-1} \varpi x\right]^{\times} \subset$ $\mathcal{K}_{\mathfrak{A}}$. Thus conjugation by $x$ can be viewed as providing another $F$-embedding of $E$ in $A$ taking $E^{\times}$ into $\mathcal{K}_{\mathfrak{A}}$. At this point, we break off to point out an elementary fact.

Exercise 5.1. Let $\mathfrak{B}$ be a hereditary $\mathfrak{o}_{F}$-order in $A$ and let $L / F$ be a subfield of $A$ with $L^{\times} \subset \mathcal{K}_{\mathfrak{B}}$. An element $x \in G$ then satisfies $x L^{\times} x^{-1} \subset \mathcal{K}_{\mathfrak{B}}$ if and only if $x \in \mathcal{K}_{\mathfrak{B}} Z_{G}\left(L^{\times}\right)$.

Hint. Let $\Lambda$ be the lattice chain attached to $\mathfrak{B}$, so that $\mathcal{K}_{\mathfrak{B}}$ is the group of $x \in G$ such that $x \Lambda=\Lambda$. In particular, $\Lambda^{\prime}=x \Lambda$ is $L^{\times}$-stable if and only if $x L^{\times} x^{-1} \subset \mathcal{K}_{\mathfrak{B}}$. On the other hand, $\Lambda^{\prime}$ is isomorphic to $\Lambda$ as $\mathfrak{o}_{F}$-lattice chain, and is $L^{\times}$-stable if and only if $\Lambda^{\prime}=y \Lambda$, for some $y \in Z_{G}\left(L^{\times}\right)$.

In the present case, $Z_{G}\left(E^{\times}\right)=E^{\times} \subset \mathcal{K}_{\mathfrak{A}}$, so $x \in \mathcal{K}_{\mathfrak{A}}$, as required. The proof of Lemma 5.1 is complete.

Since $\mathcal{K}_{\mathfrak{A}}=E^{\times} U_{\mathfrak{A}}$ and $\boldsymbol{J}=E^{\times} J^{1}$, we can rewrite the expansion (5.1.1) as

$$
\begin{aligned}
\operatorname{tr} \pi(1+\varpi) & =\sum_{x \in \mathcal{K}_{\mathfrak{A}} / J} \operatorname{tr} \Lambda\left(x^{-1}(1+\varpi) x\right) \\
& =\sum_{x \in U_{\mathfrak{A}} / J^{1}} \operatorname{tr} \eta\left(x^{-1}(1+\varpi) x\right) .
\end{aligned}
$$

In particular, the series (5.1.2) has only finitely many terms, so there are no convergence difficulties. Also, $\operatorname{tr} \eta\left(x^{-1}(1+\varpi) x\right)=0$ unless $x^{-1}(1+\varpi) x \in H^{1}$. We therefore have to determine the set of $x \in U_{\mathfrak{A}}$ for which $x^{-1}(1+\varpi) x \in H^{1}$. 


\section{J. Bushnell and G. Henniart}

5.2 Let $r_{1}<r_{2}<\cdots<r_{t}$ be the jumps of $\xi$ (or $\beta$ ). We define fields $E_{i}$ as in $\S 1.1$, and we let $A_{i}$ be the $A$-centralizer of $E_{i}$. We put $\mathfrak{A}_{i}=A_{i} \cap \mathfrak{A}$ and $\mathfrak{P}_{i}=\operatorname{rad} \mathfrak{A}_{i}$. We have

$$
\begin{gathered}
F=E_{r_{t}} \subset E_{r_{t-1}} \subset \cdots \subset E_{r_{1}} \subset E_{0}=E, \\
\mathfrak{A}=\mathfrak{A}_{r_{t}} \supset \mathfrak{A}_{r_{t-1}} \supset \cdots \supset \mathfrak{A}_{r_{1}} \supset \mathfrak{A}_{0}=\mathfrak{o}_{E}, \\
\mathfrak{P}=\mathfrak{P}_{r_{t}} \supset \mathfrak{P}_{r_{t-1}} \supset \cdots \supset \mathfrak{P}_{r_{1}} \supset \mathfrak{P}_{0}=\mathfrak{p}_{E},
\end{gathered}
$$

with all containments strict.

With this notation, Proposition 3.6 implies that the $\mathfrak{o}_{F}$-orders $\mathfrak{H}=\mathfrak{H}(\beta, \mathfrak{A})$ and $\mathfrak{J}=\mathfrak{J}(\beta, \mathfrak{A})$ of [BK93, ch. 3] are given by

$$
\mathfrak{H}=\mathfrak{o}_{E}+\sum_{i=1}^{t} \mathfrak{P}_{r_{i}}^{\left[r_{i} / 2\right]+1}, \quad \mathfrak{J}=\mathfrak{o}_{E}+\sum_{i=1}^{t} \mathfrak{P}_{r_{i}}^{\left[\left(r_{i}+1\right) / 2\right]} .
$$

(We recall that, for example, $H^{1}=1+\mathfrak{H} \cap \mathfrak{P}$.) We define a third $\mathfrak{o}_{F}$-order $\mathfrak{M}=\mathfrak{M}(\beta, \mathfrak{A})$ by

$$
\mathfrak{M}=\mathfrak{o}_{E}+\sum_{i=1}^{t} \mathfrak{P}_{r_{i}}^{\left[r_{i} / 2\right]}
$$

We define groups $M^{k}=1+\mathfrak{M} \cap \mathfrak{P}^{k}, k \geqslant 1$. In particular, we have

$$
H^{1} \subset J^{1} \subset M^{1} \subset U_{\mathfrak{A}}^{1},
$$

and $H^{1}$ and $J^{1}$ are normal subgroups of $M^{1}$.

Remark 5.2. Because of the containment relations between the $E_{r_{i}}$, these assertions are obvious. In the general situation of [BK93], where $E / F$ can be wildly ramified, considerably more effort is required even to deal with the groups $H^{1}$ and $J^{1}$.

Since $E / F$ is totally tamely ramified, the subfield $E_{i}$ of $E$ is determined by the degree $d_{i}=\left[E_{i}: F\right]$. It follows that the lattices $\mathfrak{H}, \mathfrak{J}$ and $\mathfrak{M}$ are completely determined by the field $E$ and the sequence of integers $\left\{d_{i}\right\}$.

We will need a larger group. Consider the $G$-normalizer $N_{G}\left(E_{1}^{\times}\right)$and $G$-centralizer $Z_{G}\left(E_{1}^{\times}\right)$ of the field $E_{1}$. By Exercise 5.1, we have $N_{G}\left(E_{1}^{\times}\right) \subset \mathcal{K}_{\mathfrak{A}} Z_{G}\left(E_{1}^{\times}\right)=U_{\mathfrak{A}} Z_{G}\left(E_{1}^{\times}\right)$. Thus $N_{G}\left(E_{1}^{\times}\right)=$ $N_{U_{\mathfrak{A}}}\left(E_{1}^{\times}\right) Z_{G}\left(E_{1}^{\times}\right)$.

The quotient $N_{G}\left(E_{1}^{\times}\right) / Z_{G}\left(E_{1}^{\times}\right)$is isomorphic to the group $\operatorname{Aut}\left(E_{1} \mid F\right)$ of $F$-automorphisms of the field $E_{1}$. This finite group has order prime to $p$ and $U_{\mathfrak{A}}^{1}$ is a pro- $p$-group, so

$$
\begin{gathered}
N_{G}\left(E_{1}^{\times}\right) \cap U_{\mathfrak{A}}^{1}=Z_{G}\left(E_{1}^{\times}\right) \cap U_{\mathfrak{A}}^{1} \subset H^{1}, \\
N_{U_{\mathfrak{A}}}\left(E_{1}^{\times}\right) / Z_{U_{\mathfrak{A}}}\left(E_{1}^{\times}\right) \cong \operatorname{Aut}\left(E_{1} \mid F\right) .
\end{gathered}
$$

We define

$$
M=N_{U_{\mathfrak{A}}}\left(E_{1}^{\times}\right) M^{1} .
$$

Note that, in the case $r_{1}>1$, we have $E_{1}=E$.

The group $N_{U_{\mathfrak{A}}}\left(E_{1}^{\times}\right)$normalizes each field $E_{i}, i \geqslant 1$, since $E_{i} / F$ is the unique sub-extension of $E_{1} / F$ of degree $d_{i}$. It follows that the group $M$ normalizes both $H^{1}$ and $J^{1}$. Its significance is indicated by the following proposition.

Proposition 5.2. Let $x \in U_{\mathfrak{A}}$. Then $x^{-1}(1+\varpi) x \in H^{1}$ if and only if $x \in M$.

The proof occupies the next two sections. Observe, however, that $1+\varpi \in H^{1}$ and $M$ normalizes $H^{1}$, so the sufficiency of the condition is immediate. 


\section{TAME LANGLANDS CORRESPONDENCE: TOTALLY RAMIFIED REPRESENTATIONS}

5.3 To prove Proposition 5.2, we need to first recall some machinery from [BK93, ch. 1]. Let $s_{E}: A \rightarrow E$ be the tame corestriction on $A$, relative to $E / F$, which is the identity on $E$ (see [BK93, (1.3.8)]). We let $a_{\varpi}: x \mapsto \varpi x-x \varpi$ be the adjoint map. It will also be useful to have the normalized version $\boldsymbol{a}_{\varpi}(x)=\varpi x \varpi^{-1}-x$. We have an exact sequence

$$
\ldots \stackrel{\boldsymbol{a}_{\varpi}}{\longrightarrow} A \stackrel{s_{E}}{\longrightarrow} A \stackrel{\boldsymbol{a}_{\varpi}}{\longrightarrow} A \stackrel{s_{E}}{\longrightarrow} \cdots .
$$

Lemma 5.3. Let $L$ be an $\mathfrak{o}_{E}$-lattice in $A$, invariant under conjugation by $E^{\times}$. Suppose that $s_{E}(L)=$ $L \cap E$. Then $L=L \cap \boldsymbol{a}_{\varpi}(A) \oplus L \cap E$ and the sequence

$$
\cdots \stackrel{\boldsymbol{a}_{\varpi}}{\longrightarrow} L \stackrel{s_{E}}{\longrightarrow} L \stackrel{\boldsymbol{a}_{\varpi}}{\longrightarrow} L \stackrel{s_{E}}{\longrightarrow} \cdots
$$

is exact.

Proof. The kernel of the map $s_{E}: L \rightarrow s_{E}(L)$ is $L \cap \boldsymbol{a}_{\varpi}(A)$, so we have the decomposition $L=$ $s_{E}(L) \oplus L \cap \boldsymbol{a}_{\varpi}(A)=L \cap E \oplus L \cap \boldsymbol{a}_{\varpi}(A)$. Therefore $\boldsymbol{a}_{\varpi}$ maps $L \cap \boldsymbol{a}_{\varpi}(A)$ isomorphically onto $\boldsymbol{a}_{\varpi}(L)$, that is, $\boldsymbol{a}_{\varpi}(L)=L \cap \boldsymbol{a}_{\varpi}(A)$, as required.

The hypotheses of Lemma 5.3 apply, in particular, to the lattices $\mathfrak{P}^{k}, \mathfrak{M}^{k}=\mathfrak{M} \cap \mathfrak{P}^{k}$ and $\mathfrak{H}^{k}$.

We now prove Proposition 5.2 under the additional hypothesis that $x \in U_{\mathfrak{A}}^{1}$. We have to show that $x^{-1}(1+\varpi) x \in H^{1}$ implies $x \in M^{1}$. We write $x=1+y$. Nothing is changed if we replace $y$ by $y+z$ with $z \in \mathfrak{p}_{E}$, so we may as well assume $y \in \boldsymbol{a}_{\varpi}(\mathfrak{P})$. Set $k=v_{\mathfrak{A}}(y)$ (meaning that $\left.y \in \mathfrak{P}^{k} \backslash \mathfrak{P}^{k+1}\right)$; we therefore take $y \in \boldsymbol{a}_{\varpi}\left(\mathfrak{P}^{k}\right)$. If $k \geqslant[l / 2]$, we have $x \in M^{1}$ and there is nothing to prove. We therefore assume the contrary and work by induction on the integer $k \geqslant 1$.

We write $(1+y)^{-1}=1+\bar{y}$; thus we have $y+\bar{y}+y \bar{y}=y+\bar{y}+\bar{y} y=0$. Using such elementary identities, we get

$$
\begin{aligned}
x^{-1}(1+\varpi) x & =(1+\bar{y})(1+\varpi)(1+y) \\
& =1+\bar{y}+\varpi+\bar{y} \varpi+y+\bar{y} y+\varpi y+\bar{y} \varpi y \\
& \equiv 1+\varpi+a_{\varpi}(y) \quad\left(\bmod \mathfrak{P}^{2 k+1}\right) .
\end{aligned}
$$

It follows from Lemma 5.3 that $y \in \mathfrak{M}^{k}+\mathfrak{P}^{k+1}$ and then that $x \in U_{\mathfrak{A}}^{k+1} M^{k}$. We write $x=a b$, with $a \in U_{\mathfrak{A}}^{k+1}$ and $b \in M^{k}$. Since $M^{k}$ normalizes $H^{1}$, we have $x^{-1}(1+\varpi) x \in H^{1}$ if and only if $a^{-1}(1+\varpi) a \in H^{1}$ and Proposition 5.2 follows, by induction, in this case.

5.4 To complete the proof of Proposition 5.2, we have to take $u \in U_{\mathfrak{A}}$ such that $u^{-1}(1+\varpi) u \in H^{1}$ and show that $u \in M$. We first make a simplification.

There exists a prime element $\varpi_{E}$ of $E$ such that $\varpi_{E}^{n} \in F$ and $\varpi_{E} \equiv \varpi\left(\bmod U_{E}^{1}\right)$. Suppose that the result holds for $\varpi_{E}$. Given any other prime element $\varpi$ of $E$, we can write $\varpi_{E}=\sum_{j \geqslant 1} \alpha_{j} \varpi^{j}$, with $\alpha_{j} \in \boldsymbol{\mu}_{F} \cup\{0\}$. Any element of $U_{\mathfrak{A}}$ which conjugates $1+\varpi$ into $H^{1}$ then surely conjugates $1+\varpi_{E}$ into $H^{1}$ and so lies in $M$. In other words, we may proceed on the hypothesis that $\varpi^{n}$ is a prime element of $F$. In this situation, the following holds.

LEMma 5.4. There is a subgroup $\boldsymbol{\mu}_{\mathfrak{A}}$ of $U_{\mathfrak{A}}$ satisfying these conditions:

(1) $U_{\mathfrak{A}}=\boldsymbol{\mu}_{\mathfrak{A}} \ltimes U_{\mathfrak{A}}^{1}$;

(2) $\boldsymbol{\mu}_{\mathfrak{A}}$ is stable under conjugation by $\varpi$;

(3) $\boldsymbol{\mu}_{\mathfrak{A}} \cong \boldsymbol{\mu}_{F}^{n}$, the factors $\boldsymbol{\mu}_{F}$ being permuted cyclically under conjugation by $\varpi$.

We therefore have

$$
\mathcal{K}_{\mathfrak{A}}=\left\langle\varpi, \boldsymbol{\mu}_{\mathfrak{A}}\right\rangle \ltimes U_{\mathfrak{A}}^{1}
$$




\section{J. Bushnell and G. Henniart}

We write $u=\zeta u_{1}$, with $\zeta \in \boldsymbol{\mu}_{\mathfrak{A}}$ and $u_{1} \in U_{\mathfrak{A}}^{1}$. Consider the element $u^{-1} \varpi u$ of $\mathfrak{P}$. We have

$$
u^{-1} \varpi u \equiv \zeta^{-1} \varpi \zeta \quad\left(\bmod \mathfrak{P}^{2}\right) .
$$

We have two cases:

$$
\mathfrak{H}^{1}+\mathfrak{P}^{2}= \begin{cases}\mathfrak{P}_{1}+\mathfrak{P}^{2} & \text { if } r_{1}=1, \\ \mathfrak{p}_{E}+\mathfrak{P}^{2} & \text { otherwise. }\end{cases}
$$

We set $\varpi_{1}=\varpi^{\left[E: E_{1}\right]}$ in the first case, and $\varpi_{1}=\varpi$ in the second. The element $\zeta^{-1} \varpi \zeta+\mathfrak{P}^{2}$ of $\mathfrak{P} / \mathfrak{P}^{2}$ lies in the image of $\mathfrak{H}^{1}$ if and only if it commutes with $\varpi_{1}$ : this follows from Lemma 5.3. However, $\varpi_{1}$ and $\zeta^{-1} \varpi \zeta$ both lie in the group $\left\langle\varpi, \boldsymbol{\mu}_{\mathfrak{A}}\right\rangle \cong \mathbb{Z} \ltimes \boldsymbol{\mu}_{\mathfrak{A}}$; they commute if and only if their images in $\mathcal{K}_{\mathfrak{A}} / U_{\mathfrak{A}}^{1}$ commute. In the second case, this is equivalent to $\zeta^{-1} \varpi \zeta \in \varpi \boldsymbol{\mu}_{F}$, or $\zeta \in M$. In the first case, $\zeta^{-1} \varpi \zeta$ commutes with $\varpi_{1}$ if and only if $\zeta \varpi_{1} \zeta^{-1}$ commutes with $\varpi$; this is equivalent to $\zeta \varpi_{1} \zeta^{-1} \in E$, that is, $\zeta \varpi_{1} \zeta^{-1} \in E_{1}$. This is equivalent to demanding $\zeta \in M$.

Thus the condition $\zeta^{-1} u_{1}^{-1}(1+\varpi) u_{1} \zeta \in H^{1}$ implies $\zeta \in M$ and hence

$$
u_{1}^{-1}(1+\varpi) u_{1} \in H^{1} .
$$

We proved in $\S 5.3$ that this implies $u_{1} \in M^{1}$, so we have completed the proof of Proposition 5.2.

5.5 As in $\S 5.4$, we can write $M=\boldsymbol{\mu}_{M} \ltimes M^{1}$, for a certain subgroup $\boldsymbol{\mu}_{M}$ of $\boldsymbol{\mu}_{\mathfrak{A}}$ : indeed, $\boldsymbol{\mu}_{M}$ is the $\boldsymbol{\mu}_{\mathfrak{A}}$-normalizer of $E_{1}^{\times}$. We note that $J=\boldsymbol{\mu}_{F} \times J^{1}$. Thus, writing $\theta^{\tau}$ for the character $h \mapsto \theta\left(\tau h \tau^{-1}\right)$ of $H^{1}$ and with the commutator convention $[x, y]=x^{-1} y^{-1} x y$, we have

$$
\operatorname{tr} \pi(1+\varpi)=\operatorname{dim} \Lambda \sum_{\tau \in \boldsymbol{\mu}_{M} / \boldsymbol{\mu}_{F}} \theta^{\tau}(1+\varpi) \sum_{u \in M^{1} / J^{1}} \theta^{\tau}[1+\varpi, u] .
$$

We can expand further.

Theorem 5.5. Suppose that $p \neq 2$ and let $\varpi$ be a prime element of $E$. There is a non-zero constant $\mathfrak{G}_{F}\left(\varpi, \psi_{F}\right)$ such that

$$
\operatorname{tr} \pi(1+\varpi)=\mathfrak{G}_{F}\left(\varpi, \psi_{F}\right) \operatorname{dim} \Lambda \sum_{\tau \in \boldsymbol{\mu}_{M} / \boldsymbol{\mu}_{F}} \varepsilon(\tau, \varpi) \theta^{\tau}(1+\varpi),
$$

where $\varepsilon(\tau, \varpi)= \pm 1$ and $\varepsilon(1, \varpi)=1$.

Remark 5.5.1. We shall specify the constant $\mathfrak{G}_{F}\left(\varpi, \psi_{F}\right)$ in the course of the proof ( $\$ 5.7$ below). It is a product of classical quadratic Gauss sums and hence, since $p \neq 2$, it is non-zero. The case $p=2$ would require further analysis, for which we will have no direct need: we therefore exclude it. The signs $\varepsilon(\tau, \varpi)$ are of no enduring interest.

Remark 5.5.2. Let $\varkappa$ be a tamely ramified character of $F^{\times}$which is trivial on $\mathrm{N}_{E / F}\left(E^{\times}\right)$. Thus $\varkappa \circ$ det is trivial on $\boldsymbol{J}$, and we may form the $\varkappa$-twisted trace of $\pi$,

$$
\Xi_{\pi}^{\varkappa}(g)=\sum_{x \in G / \boldsymbol{J}} \varkappa(\operatorname{det} x)^{-1} \operatorname{tr} \Lambda\left(x^{-1} g x\right),
$$

for suitable elements $g \in G$ (see [BH96, $\S 15.8$ and Appendix]). An argument identical to the proof of the theorem yields

$$
\Xi_{\pi}^{\varkappa}(1+\varpi)=\mathfrak{G}_{F}\left(\varpi, \psi_{F}\right) \operatorname{dim} \Lambda \sum_{\tau \in \boldsymbol{\mu}_{M} / \boldsymbol{\mu}_{F}} \varkappa(\operatorname{det} \tau) \varepsilon(\tau, \varpi) \theta^{\tau}(1+\varpi),
$$

with the same signs $\varepsilon(\tau, \varpi)$ as before. 


\section{TAME LANGLANDS CORRESPONDENCE: TOTALLY RAMIFIED REPRESENTATIONS}

5.6 As the first step in the proof of Theorem 5.5, we have the following proposition.

Proposition 5.6. Let $S$ denote the set of integers $i$ for which $r_{i}$ is an odd jump of $\xi$ and $r_{i} \geqslant 3$. Set $r_{i}=2 s_{i}+1, i \in S$. We have

$$
\operatorname{tr} \pi(1+\varpi)=\operatorname{dim} \Lambda \sum_{\tau \in \boldsymbol{\mu}_{M} / \boldsymbol{\mu}_{F}} \theta^{\tau}(1+\varpi) \prod_{i} \sum_{y_{i}} \theta^{\tau}\left[1+\varpi, 1+y_{i}\right],
$$

where $i$ ranges over $S$ and $1+y_{i}$ over $M^{s_{i}} / J^{s_{i}} M^{s_{i}+1}$.

Proof. We note here that, for $\tau \in \boldsymbol{\mu}_{M},\left[\mathfrak{A}, l, 0, \beta^{\tau}\right]$ is a simple stratum and $\theta^{\tau} \in \mathcal{C}\left(\mathfrak{A}, \beta^{\tau}, \psi_{F}\right)$, while $H^{1}\left(\beta^{\tau}, \mathfrak{A}\right)=H^{1}(\beta, \mathfrak{A})^{\tau}=H^{1}$, since $\tau \in M$. Moreover, $\beta^{\tau}$ has the same jumps as $\beta$.

For the rest of the proof, we use the symbol $\vartheta$ to denote any of the characters $\theta^{\tau}$. Every coset of $M^{1} / J^{1}$ contains an element $\prod_{i \in S}\left(1+y_{i}\right)$, with $y_{i} \in \mathfrak{P}_{r_{i}}^{s_{i}}$. We expand the commutator

$$
\left[1+\varpi, \prod_{i \in S}\left(1+y_{i}\right)\right]
$$

in the standard way, using the rule $[a, b c]=[a, c] \cdot c^{-1}[a, b] c$. The element $\left(1+y_{i}\right)$ stabilizes $\vartheta$ on $H^{s_{j}+1}$, for $j>i$, by [BK93, (3.3.2)], so we have

$$
\vartheta[1+\varpi, 1+y]=\prod_{i \in S} \vartheta\left[1+\varpi, 1+y_{i}\right]
$$

We deduce that

$$
\sum_{1+y \in M^{1} / J^{1}} \vartheta[1+\varpi, 1+y]=\prod_{i \in S} \sum_{y_{i}} \vartheta\left[1+\varpi, 1+y_{i}\right],
$$

where $1+y_{i}$ ranges over $M^{s_{i}} / J^{s_{i}} M^{s_{i}+1}$, and the result follows.

5.7 We make a preliminary evaluation of the innermost terms of (5.6.1). As before, if $r_{i} \geqslant 3$ is an odd jump of $\xi$, we take a simple stratum $\left[\mathfrak{A}, l, r_{i}, \gamma_{r_{i}}\right]$ equivalent to $\left[\mathfrak{A}, l, r_{i}, \beta\right]$ with $F\left[\gamma_{r_{i}}\right]=E_{r_{i}}$ (see $\S$ 3.6). We choose a simple stratum $\left[\mathfrak{A}_{i}, r_{i}, r_{i}-1, \alpha_{i}\right]$ equivalent to $\left[\mathfrak{A}_{i}, r_{i}, r_{i}-1, \beta-\gamma_{r_{i}}\right]$ with $F\left[\gamma_{i}, \alpha_{i}\right]=E_{r_{i}-1}$.

We write $\psi_{A}=\psi_{F} \circ \operatorname{tr}_{A}$, where $\operatorname{tr}_{A}: A \rightarrow F$ is the trace map.

LemMA 5.7.1. Let $i \in S$ and $\tau \in \boldsymbol{\mu}_{M}$. We have

$$
\theta^{\tau}[1+\varpi, 1+y]=\psi_{A}\left(\alpha_{i}^{\tau} a_{\varpi}(y) y\right), \quad y \in \mathfrak{M}^{s_{i}} .
$$

Proof. Let $y \in \mathfrak{M}^{s_{i}}$. The commutator $[1+\varpi, 1+y]$ lies in $H^{s_{i}+1}$ and so, directly from the definition of simple character in $[\mathrm{BK} 93,(3.2)]$, we get

$$
\theta[1+\varpi, 1+y]=\psi_{A}\left(\alpha_{i}([1+\varpi, 1+y]-1)\right) .
$$

The right-hand side of (5.7.2) depends only on the coset $[1+\varpi, 1+y] U_{\mathfrak{A}}^{r_{i}+1}$, since $\alpha_{i} \in \mathfrak{P}^{-r_{i}}$. We expand the commutator, using the notation $(1+y)^{-1}=1+\bar{y}$. Thus, for example, $y+\bar{y}+\bar{y} y=0$. We obtain

$$
[1+\varpi, 1+y] \equiv 1+\bar{\varpi} \bar{y}+\bar{y} \varpi+\bar{\varpi} \bar{y} \varpi+\bar{\varpi} \bar{y} y+\bar{y} \varpi y \quad\left(\bmod U_{\mathfrak{A}}^{r_{i}+1}\right) .
$$

As $\alpha_{i}$ commutes with $\varpi$ and $\bar{\varpi}$, this reduces to $\theta[1+\varpi, 1+y]=\psi_{A}\left(\alpha_{i} a_{\varpi}(y) y\right)$.

We now replace $\theta$ by $\theta^{\tau}, \tau \in \boldsymbol{\mu}_{M}$. This has the effect of replacing $\alpha_{i}$ by $\alpha_{i}^{\tau}$ in (5.7.2). Since $r_{i} \geqslant 3$, the element $\alpha_{i}^{\tau}$ lies in $E_{1} \subset E$ and so it commutes with $\varpi$. We can therefore use the same calculation to get the desired result.

We may usefully re-interpret the formula (5.7.1). Taking $i \in S$ as before, the abelian group $M^{s_{i}} / J^{s_{i}} M^{s_{i}+1}$ is a $\Gamma$-module, where $\Gamma=E^{\times} / F^{\times} U_{E}^{1}$. In the notation of $\S 4.2$, we have our next lemma. 


\section{J. Bushnell and G. Henniart}

Lemma 5.7.2. Let $i \in S$ and let $\varpi$ be a prime element of $E$. The map $1+y \mapsto \varpi^{-s_{i}} y$ induces a Г-isomorphism

$$
M^{s_{i}} / J^{s_{i}} M^{s_{i}+1} \cong I_{\mathfrak{A}}\left(n / d_{r_{i}-1}, n / d_{r_{i}}\right) .
$$

It will be easier to write

$$
\mathcal{M}_{i}=I_{\mathfrak{A}}\left(n / d_{r_{i}-1}, n / d_{r_{i}}\right), \quad i \in S .
$$

Consider the function on $\mathcal{M}_{i}$ given by (5.7.1),

$$
x \longmapsto \theta^{\tau}\left[1+\varpi, 1+\varpi^{s_{i}} x\right] .
$$

If we write $\alpha_{i}^{\tau} \equiv \zeta_{i}(\varpi, \tau) \varpi^{-r_{i}}\left(\bmod U_{E}^{1}\right)$, for some $\zeta_{i}(\varpi, \tau) \in \boldsymbol{\mu}_{F}$, this reduces to

$$
x \longmapsto \psi_{F}\left(\zeta_{i}(\varpi, \tau) \boldsymbol{q}_{F}^{(i)}(x)\right),
$$

where $\boldsymbol{q}_{F}^{(i)}: \mathcal{M}_{i} \rightarrow \mathbb{k}_{F}$ denotes the $\Gamma$-invariant quadratic form

$$
x \longmapsto \operatorname{tr}_{A}\left(\left(x-\varpi^{-1} x \varpi\right) \cdot \varpi^{s_{i}} x \varpi^{-s_{i}}\right) \quad\left(\bmod \mathfrak{p}_{F}\right) .
$$

As in $\S 4.5$, we write

$$
\mathfrak{g}\left(\zeta \boldsymbol{q}_{F}^{(i)}, \psi_{F}\right)=\sum_{x \in \mathcal{M}_{i}} \psi_{F}\left(\zeta \boldsymbol{q}_{F}^{(i)}(x)\right), \quad \zeta \in \boldsymbol{\mu}_{F} .
$$

This is just a classical quadratic Gauss sum and, since $p \neq 2$, it is non-zero. We further have the relation

$$
\mathfrak{g}\left(\zeta \boldsymbol{q}_{F}^{(i)}, \psi_{F}\right)=\left(\frac{\zeta}{q}\right)^{t} \mathfrak{g}\left(\boldsymbol{q}_{F}^{(i)}, \psi_{F}\right), \quad \zeta \in \boldsymbol{\mu}_{F},
$$

where $t$ is the rank of $\boldsymbol{q}_{F}^{(i)}$. To summarize, we can write all this as follows.

Proposition 5.7. Let $i \in S$, and suppose $p \neq 2$.

(1) There is a unique $\zeta_{i}(\varpi) \in \boldsymbol{\mu}_{F}$ such that $\alpha_{i} \varpi^{r_{i}} \equiv \zeta_{i}(\varpi)\left(\bmod U_{E}^{1}\right)$.

(2) There is a $\Gamma$-invariant quadratic form $\boldsymbol{q}_{F}^{(i)}: \mathcal{M}_{i} \rightarrow \mathbb{k}_{F}$ such that

$$
\theta\left[1+\varpi, 1+\varpi^{s_{i}} x\right]=\psi_{F}\left(\zeta_{i}(\varpi) \boldsymbol{q}_{F}^{(i)}(x)\right), \quad x \in \mathcal{M}_{i} .
$$

In particular,

$$
\sum_{y \in M^{s_{i}} / J^{s_{i}} M^{s_{i}+1}} \theta[1+\varpi, y]=\mathfrak{g}\left(\zeta_{i}(\varpi) \boldsymbol{q}_{F}^{(i)}, \psi_{F}\right),
$$

and this quantity is non-zero.

(3) Let $\tau \in \boldsymbol{\mu}_{M}$. There are constants $\varepsilon_{i}(\tau, \varpi)= \pm 1$, with $\varepsilon_{i}(1, \varpi)=1$, such that

$$
\sum_{y \in M^{s_{i}} / J^{s_{i}} M^{s_{i}+1}} \theta^{\tau}[1+\varpi, y]=\varepsilon_{i}(\tau, \varpi) \mathfrak{g}\left(\zeta_{i}(\varpi) \boldsymbol{q}_{F}^{(i)}, \psi_{F}\right) .
$$

We extend our notation by setting

$$
\mathfrak{G}_{F}\left(\varpi, \psi_{F}\right)=\prod_{i \in S} \mathfrak{g}\left(\zeta_{i}(\varpi) \boldsymbol{q}_{F}^{(i)}, \psi_{F}\right), \quad \varepsilon(\tau, \varpi)=\prod_{i \in S} \varepsilon_{i}(\tau, \varpi) .
$$

We then have

$$
\sum_{y \in M^{1} / J^{1}} \theta^{\tau}[1+\varpi, y]=\varepsilon(\tau, \varpi) \mathfrak{G}_{F}\left(\varpi, \psi_{F}\right)
$$




\section{TAME LANGLANDS CORRESPONDENCE: TOTALLY RAMIFIED REPRESENTATIONS}

and

$$
\operatorname{tr} \pi(1+\varpi)=\operatorname{dim} \Lambda \cdot \mathfrak{G}_{F}\left(\varpi, \psi_{F}\right) \sum_{\tau \in \boldsymbol{\mu}_{M} / \boldsymbol{\mu}_{F}} \varepsilon(\tau, \varpi) \theta^{\tau}(1+\varpi),
$$

as required for Theorem 5.5.

Remark 5.7. We note that the expression (5.5.2) for the function $\varpi \mapsto \operatorname{tr} \pi(1+\varpi)$ depends only on the element $\beta$ or, equivalently, on $\xi \mid U_{E}^{1}$. The same applies to the twisted trace formula (5.5.3).

\section{Automorphic induction}

We continue in the situation of $\S 5$, but we introduce a further element of structure. We let $K / F$ be a cyclic sub-extension of $E / F$ of prime degree $d$. (This restriction is not necessary for much of the time; we are, moreover, only really interested in the case $d=2$.) The pair $(E / K, \xi)$ is then admissible, so we can form the representation $\rho={ }_{K} \pi_{\xi} \in \mathcal{A}_{n / d}^{\text {et }}(K)$. We write $\Sigma=\operatorname{Gal}(K / F)$, and we choose a character $\varkappa$ of $F^{\times}$which generates the group of characters of $F^{\times}$which are trivial on $\mathrm{N}_{K / F}\left(K^{\times}\right)$.

6.1 We recall that we regard the field $E$ as embedded in $A=\mathrm{M}_{n}(F)$; we let $A_{K}$ denote the $A$-centralizer of $K$ and put $G_{K}=A_{K}^{\times}$. We set $\mathfrak{A}_{K}=\mathfrak{A} \cap A_{K}$. The quadruple $\left[\mathfrak{A}_{K}, l, 0, \beta\right]$ is then a simple stratum in $A_{K}$. We have $H_{K}^{1}=H^{1}\left(\beta, \mathfrak{A}_{K}\right)=H^{1} \cap G_{K}$, and so on [BH96]. The character $\theta_{K}=\theta \mid H_{K}^{1}$ lies in $\mathcal{C}\left(\mathfrak{A}_{K}, \beta, \psi_{K}\right)$, where $\psi_{K}=\psi_{F} \circ \operatorname{Tr}_{K / F}$, and $\theta_{K}$ occurs in $\rho$. Indeed, there is a unique irreducible representation $\Lambda_{K}$ of the group $\boldsymbol{J}_{K}=E^{\times} J_{K}^{1}$ such that $\Lambda_{K} \mid H_{K}^{1}$ is a multiple of $\theta_{K}$ and $\rho \cong c$-Ind $\Lambda_{K}$.

We define subgroups $M_{K}^{1} \subset M_{K} \subset G_{K}$, exactly as in $\S 5.2$. We observe that any jump of $\beta$ over $K$ is a jump of $\beta$ over $F$. On the other hand, since $d$ is prime, there exists at most one jump of $\beta$ over $F$ which is not a jump of $\beta$ over $K$.

Lemma 6.1. Suppose that $K \subset E_{1}$. Let $\sigma \in \Sigma$, and suppose that $\operatorname{tr} \rho^{\sigma}(1+\varpi) \neq 0$, for some prime element $\varpi$ of $E$. Then $\sigma$ extends to an automorphism of $E_{1} / F$.

Proof. Recall that $r_{1}$ denotes the least jump of $\beta$ over $F$. Thus $r_{1} \geqslant 1$, while $r_{1} \geqslant 2$ if and only if $E_{1}=E$.

Suppose first that $r_{1} \geqslant 2$. We choose $t_{\sigma} \in G$ such that $\sigma(h)=t_{\sigma} h t_{\sigma}^{-1}, h \in G_{K}$. If $1+\sigma(\varpi)$ lies in the support of $\operatorname{tr} \rho$, there exists $x \in G_{K}$ such that the element $h=x t_{\sigma}(1+\varpi) t_{\sigma}^{-1} x^{-1}$ lies in $\boldsymbol{J}_{K}$. Since $h$ is pro-unipotent, it lies in $J_{K}^{1}$ and in the support of $\operatorname{tr} \Lambda_{K}$, whence it lies in $H_{K}^{1}$. In particular, we have $h \in H^{1}$ so, by Proposition 5.2, we have $x t_{\sigma} \in E^{\times} M=N_{G}\left(E^{\times}\right) M^{1}$. However, by definition, $x t_{\sigma} \in N_{G}\left(K^{\times}\right)$, whence $x t_{\sigma} \in N_{G}\left(E^{\times}\right) M^{1} \cap N_{G}\left(K^{\times}\right)=N_{G}\left(E^{\times}\right)\left(M^{1} \cap N_{G}\left(K^{\times}\right)\right)$. The group $N_{G}\left(K^{\times}\right) / G_{K}=\Sigma$ has finite order prime to $p$ and $M^{1}$ is a pro- $p$-group; thus $M^{1} \cap N_{G}\left(K^{\times}\right)=$ $M^{1} \cap G_{K}=M_{K}^{1}$ and $x t_{\sigma} \in N_{G}\left(E^{\times}\right) M_{K}^{1}$. The coset $M_{K}^{1} x t_{\sigma}$ thus contains an element of $N_{G}\left(E^{\times}\right)$, and the lemma follows in this case.

Suppose that $r_{1}=1$. The same argument as before gives us

$$
x t_{\sigma} \in E^{\times} N_{U_{\mathfrak{A}}}\left(E_{1}^{\times}\right) M^{1} \cap N_{G}\left(K^{\times}\right)=E^{\times}\left(N_{U_{\mathfrak{A}}}\left(E_{1}^{\times}\right) \cap N_{G}\left(K^{\times}\right)\right) M_{K}^{1} .
$$

As $K \subset E_{1}$, the normalizer of $E_{1}$ is contained in that of $K$ and, since $K / F$ is Galois, the lemma follows.

Remark 6.1. When $K \not \subset E_{1}$, we have $K \cap E_{1}=F$ since $d=[K: F]$ is prime. Any $F$-automorphism of $K$ extends by triviality to one of $E_{1}$. 


\section{J. Bushnell and G. Henniart}

6.2 By Theorem 5.5, we have

$$
\operatorname{tr} \rho(1+\varpi)=\mathfrak{G}_{K}\left(\varpi, \psi_{K}\right) \operatorname{dim} \Lambda_{K} \sum_{\tau \in \boldsymbol{\mu}_{M_{K}} / \boldsymbol{\mu}_{F}} \varepsilon_{K}(\tau, \varpi) \theta^{\tau}(1+\varpi),
$$

for signs $\varepsilon_{K}(\tau, \varpi)$ with $\varepsilon_{K}(1, \varpi)=1$, and a constant $\mathfrak{G}_{K}\left(\varpi, \psi_{K}\right)$ defined as in $\S 5.7$. We apply Lemma 6.1 to get

$$
\sum_{\sigma \in \Sigma} \operatorname{tr} \rho^{\sigma}(1+\varpi)=\mathfrak{G}_{K}\left(\varpi, \psi_{K}\right) \operatorname{dim} \Lambda_{K} \sum_{\tau \in \boldsymbol{\mu}_{M} / \boldsymbol{\mu}_{F}} \varepsilon_{K}(\tau, \varpi) \theta^{\tau}(1+\varpi),
$$

for various signs $\varepsilon_{K}(\tau, \varpi)$.

6.3 We set $\pi=\mathrm{A}_{K / F} \rho={ }_{F} \pi_{\mu \xi}$, where $\mu={ }_{K / F} \mu_{\xi}$. The expression (5.5.3) gives the value of the $\varkappa$-trace $\Xi_{\pi}^{\varkappa}(1+\varpi)$ (cf. Remark 5.7). We apply the automorphic induction equation

$$
\Xi_{\pi}^{\varkappa}(1+\varpi)=c \boldsymbol{\delta}(1+\varpi) \sum_{\sigma \in \Sigma} \operatorname{tr} \rho^{\sigma}(1+\varpi)
$$

and (6.2.1) to get

$$
\begin{aligned}
\mathfrak{G}_{F}\left(\varpi, \psi_{F}\right) \operatorname{dim} \Lambda & \sum_{\tau \in \boldsymbol{\mu}_{M} / \boldsymbol{\mu}_{F}} \varepsilon(\tau, \varpi) \varkappa(\operatorname{det} \tau) \theta^{\tau}(1+\varpi) \\
& =c \boldsymbol{\delta}(1+\varpi) \mathfrak{G}_{K}\left(\varpi, \psi_{K}\right) \operatorname{dim} \Lambda_{K} \sum_{\tau \in \boldsymbol{\mu}_{M} / \boldsymbol{\mu}_{F}} \varepsilon_{K}(\tau, \varpi) \theta^{\tau}(1+\varpi) .
\end{aligned}
$$

6.4 We give the first of two useful simplifications. In this section, we assume that $r_{1}>1$, so that $E_{1}=E$ and $\boldsymbol{\mu}_{M} / \boldsymbol{\mu}_{F}=\operatorname{Aut}(E \mid F)$.

Proposition 6.4. Suppose that $r_{1}>1$. We then have

$$
\mathfrak{G}_{F}\left(\varpi, \psi_{F}\right) \operatorname{dim} \Lambda=c \boldsymbol{\delta}(1+\varpi) \mathfrak{G}_{K}\left(\varpi, \psi_{K}\right) \operatorname{dim} \Lambda_{K} .
$$

Proof. Let $u \in U_{E}^{2}$; we then have $(1+\varpi) u=1+\varpi v$, for some $v \in U_{E}^{1}$. From the definition, we have $\varepsilon(\tau, \varpi v)=\varepsilon(\tau, \varpi)$ for all $\tau$, and similarly for the base field $K$; likewise for the factors $\mathfrak{G}_{F}$ and $\mathfrak{G}_{K}$. Further, directly from the definition of the transfer factor, $\boldsymbol{\delta}(1+\varpi v)=k \boldsymbol{\delta}(\varpi v)=k \boldsymbol{\delta}(\varpi)$ for some positive constant $k$.

Since $r_{1}>1$, the admissibility of $(E / F, \xi)$ implies that the characters $\xi^{\alpha}, \alpha \in \operatorname{Aut}(E \mid F)$, are distinct on $U_{E}^{2}$ (cf. [BH05a, Lemma A.1]). In the identity of $\S 6.3$, we may therefore replace $1+\varpi$ by $(1+\varpi) u$, multiply by $\xi(u)^{-1}=\theta(u)^{-1}$, and integrate over $U_{E}^{2}$ to get the result.

6.5 Let us now assume $r_{1}=1$. We may therefore write $\theta=\theta_{1} \psi_{\alpha_{1}}$, where $\psi_{\alpha_{1}}$ denotes the function $1+x \mapsto \psi_{F}\left(\operatorname{tr}_{A}\left(\alpha_{1} x\right)\right)$ and $\theta_{1} \in \mathcal{C}\left(\mathfrak{A}, \gamma_{1}, \psi_{F}\right)$. Let $\boldsymbol{\mu}_{1}$ denote the $\boldsymbol{\mu}_{M}$-centralizer of $E_{1}$. Since $E_{1}=F\left[\gamma_{1}\right]$, we have $\theta_{1}^{\tau}=\theta_{1}$, and hence $\theta^{\tau}(1+\varpi)=\theta(1+\varpi) \psi_{\alpha_{1}}[\tau, 1+\varpi]$, for $\tau \in \boldsymbol{\mu}_{1} / \boldsymbol{\mu}_{F}$.

Proposition 6.5. Suppose that $r_{1}=1$, and let $\boldsymbol{\mu}_{1}$ denote the $\boldsymbol{\mu}_{M}$-centralizer of $E_{1}$. Then:

(1) $\varepsilon(\tau, \varpi)=\varepsilon_{K}(\tau, \varpi)=1$, for every $\tau \in \boldsymbol{\mu}_{1}$;

(2) the characters $\psi_{\alpha_{1}}^{\tau}, \tau \in \boldsymbol{\mu}_{1} / \boldsymbol{\mu}_{F}$, are distinct on $H^{1}$;

(3) the characters $\xi^{\tau} \mid U_{E}^{2}, \tau \in \boldsymbol{\mu}_{M} / \boldsymbol{\mu}_{1}$, are distinct; 


\section{TAME LANGLANDS CORRESPONDENCE: TOTALLY RAMIFIED REPRESENTATIONS}

(4) we have

$$
\begin{aligned}
\mathfrak{G}_{F}\left(\varpi, \psi_{F}\right) \operatorname{dim} \Lambda & \sum_{\tau \in \boldsymbol{\mu}_{1} / \boldsymbol{\mu}_{F}} \varkappa(\operatorname{det} \tau) \psi_{\alpha_{1}}[\tau, 1+\varpi] \\
& =c \boldsymbol{\delta}(1+\varpi) \mathfrak{G}_{K}\left(\varpi, \psi_{K}\right) \operatorname{dim} \Lambda_{K} \sum_{\tau \in \boldsymbol{\mu}_{1} / \boldsymbol{\mu}_{F}} \psi_{\alpha_{1}}[\tau, 1+\varpi] .
\end{aligned}
$$

Proof. Since $\tau \in \boldsymbol{\mu}_{1}$ commutes with $E_{1}$, it commutes with every $\alpha_{i}, i \in S$. The first assertion follows from the definition. The second assertion follows from the admissibility of $(E / F, \xi)$, as does the third. The fourth is then given by an argument identical to that of Proposition 6.4.

6.6 In light of Proposition 6.5, we introduce some new notation in the case $r_{1}=1$ :

$$
\begin{aligned}
\mathfrak{K}_{F}\left(\alpha_{1}, \varpi, \psi_{F}\right) & =\sum_{\tau \in \boldsymbol{\mu}_{1} / \boldsymbol{\mu}_{F}} \psi_{\alpha_{1}}[\tau, 1+\varpi], \\
\mathfrak{K}_{F}^{\varkappa}\left(\alpha_{1}, \varpi, \psi_{F}\right) & =\sum_{\tau \in \boldsymbol{\mu}_{1} / \boldsymbol{\mu}_{F}} \varkappa(\operatorname{det} \tau) \psi_{\alpha_{1}}[\tau, 1+\varpi] .
\end{aligned}
$$

Remark 6.6. When one writes them out explicitly, one finds that the quantities $\mathfrak{K}_{F}$ and $\mathfrak{K}_{F}^{\varkappa}$ are Kloosterman or Salié sums, analogous to those considered in [Duk92] and [Sal32].

Proposition 6.6. Suppose that $r_{1}=1$ and $p \neq 2$; the quantities $\mathfrak{K}_{F}\left(\alpha_{1}, \varpi, \psi_{F}\right)$ and $\mathfrak{K}_{F}^{\varkappa}\left(\alpha_{1}, \varpi, \psi_{F}\right)$ are then both non-zero and

$$
\mathfrak{K}_{F}^{\varkappa}\left(\alpha_{1}, \varpi, \psi_{F}\right) \mathfrak{G}_{F}\left(\varpi, \psi_{F}\right) \operatorname{dim} \Lambda=c \boldsymbol{\delta}(1+\varpi) \mathfrak{K}_{F}\left(\alpha_{1}, \varpi, \psi_{F}\right) \mathfrak{G}_{K}\left(\varpi, \psi_{K}\right) \operatorname{dim} \Lambda_{K} .
$$

Proof. The identity (6.6.1) follows from Proposition 6.5. Since $p \neq 2$, the factors $\mathfrak{G}_{F}$ and $\mathfrak{G}_{K}$ are non-zero.

The quantities $\psi_{\alpha_{1}}[\tau, 1+\varpi]$ are $p$ th roots of unity, while the order of $\boldsymbol{\mu}_{1} / \boldsymbol{\mu}_{F}$ divides $(q-1)^{n}$. Thus, if $\wp$ denotes the prime divisor of $p$ in the field $\mathbb{Q}\left[1^{1 / p}\right]$ of $p$ th roots of unity, we have

$$
\mathfrak{K}_{F}\left(\alpha_{1}, \varpi, \psi_{F}\right) \equiv\left|\boldsymbol{\mu}_{1} / \boldsymbol{\mu}_{F}\right| \not \equiv 0 \quad(\bmod \wp) .
$$

Therefore, $\mathfrak{K}_{F}\left(\alpha_{1}, \varpi, \psi_{F}\right) \neq 0$, and the result follows from Proposition 6.5.

We can now sum up, to get the main result of the section.

Theorem 6.6. Assume that $p \neq 2$. There are positive constants $k$ such that

$$
K / F \mu_{\xi}(\varpi)= \begin{cases}\epsilon_{F} \epsilon_{K} \frac{\mathfrak{G}_{F}\left(\varpi, \psi_{F}\right)}{\mathfrak{G}_{K}\left(\varpi, \psi_{F}\right)} k & \text { if } r_{1}>1, \\ \epsilon_{F} \epsilon_{K} \frac{\mathfrak{G}_{F}\left(\varpi, \psi_{F}\right)}{\mathfrak{G}_{K}\left(\varpi, \psi_{F}\right)} \frac{\mathfrak{K}_{F}^{\varkappa}\left(\alpha_{1}, \varpi, \psi_{F}\right)}{\mathfrak{K}_{F}\left(\alpha_{1}, \varpi, \psi_{F}\right)} k & \text { if } r_{1}=1,\end{cases}
$$

for every prime element $\varpi$ of $E$.

Proof. From [BH05a, Theorem 4.3], we have $K_{K / F} \mu_{\xi}(\varpi)=\epsilon_{F} \epsilon_{K} c \boldsymbol{\delta}(\varpi)$. We have noted that $\boldsymbol{\delta}(1+\varpi)=k \boldsymbol{\delta}(\varpi)$, for some $k>0$. The theorem now follows from Propositions 6.4 and 6.6.

To prove Theorems 3.1 and 3.2, we have to evaluate the expressions appearing in the preceding theorem. This we do in the following sections. 


\section{J. Bushnell and G. Henniart}

\section{Glauberman signs}

We compute the product $\epsilon_{F} \epsilon_{K}$ of signs occurring in Theorem 6.6. We use the notation introduced in the opening paragraphs of $\S \S 5$ and 6 . In particular, $\Gamma=E^{\times} / F^{\times} U_{E}^{1}$, and we assume throughout that $d=[K: F]=2$. (Thus $p \neq 2$.)

7.1 We write $\mathcal{J}=J^{1} / H^{1}$; thus $\mathcal{J}$ is a finite-dimensional $\mathbb{F}_{p}$-vector space and it carries the nondegenerate alternating form $h_{\theta}$ induced by $(x, y) \mapsto \theta[x, y], x, y \in J^{1}$. This form is invariant under the conjugation action of $\Gamma$ on $\mathcal{J}$ and so $\left(\mathcal{J}, h_{\theta}\right)$ provides a symplectic representation of $\Gamma$ over $\mathbb{F}_{p}$. Moreover, $\Gamma$ has only the trivial fixed point in $\mathcal{J}$. From $\S 4$.8, we have $\epsilon_{F}=t_{\Gamma}(\mathcal{J})$, so we can apply the machinery of $\S 4.7$; similarly over $K$.

We now collect some basic facts.

LEMMA 7.1.

(1) The natural inclusion of $J_{K}^{1}$ in $J^{1}$ induces a $\Gamma$-embedding $\mathcal{J}_{K} \rightarrow \mathcal{J}$ which identifies $\mathcal{J}_{K}$ with the set of $K^{\times}$-fixed points in $\mathcal{J}$.

(2) The restriction of $h_{\theta}$ to $\mathcal{J}_{K}$ is equal to $h_{\theta_{K}}$ and is, in particular, non-degenerate. Thus

$$
\mathcal{J}=\mathcal{J}_{K} \perp \mathcal{K},
$$

for a uniquely determined symplectic $\mathbb{F}_{p} \Gamma$-submodule $\mathcal{K}$ of $\mathcal{J}$.

(3) The group $K^{\times}$acts on $\mathcal{J}$ as the unique subgroup $\Gamma_{2}$ of $\Gamma$ of order 2 , and $\mathcal{K}$ is the set of all $x \in \mathcal{J}$ such that $\varpi_{K} x \varpi_{K}^{-1}=-x$, where $\varpi_{K}$ is a prime element of $K$.

For the first assertion, see [BH96]; the others are immediate.

The invariant $t_{\Gamma}$ is additive with respect to orthogonal sums, by definition; therefore Proposition 4.8 yields $\epsilon_{F} \epsilon_{K}=t_{\Gamma}(\mathcal{K})$.

7.2 For each integer $j \geqslant 1$, let $\mathcal{V}^{j}$ denote the image of $J^{j}$ in $\mathcal{J}$. From [BH05b, $\S 6.1$ ], the subspace $\mathcal{V}^{j}$ is non-degenerate. Thus there is a unique non-degenerate $\Gamma$-subspace $\mathcal{J}^{j}$ of $\mathcal{J}$ such that $\mathcal{V}^{j}=$ $\mathcal{J}^{j} \perp \mathcal{V}^{j+1}$. We have the following lemma.

LEMMA 7.2.

(1) The space $\mathcal{J}^{j}$ is zero unless $2 j$ is a jump of $\beta$.

(2) Let $j=2 s$ be an even jump of $\beta$. The quotient map $\mathcal{V}^{s} \rightarrow \mathcal{V}^{s} / \mathcal{V}^{s+1}$ induces a $\Gamma$-isomorphism $J^{s} / H^{s} J^{s+1} \cong \mathcal{J}^{s}$. We have a $\Gamma$-isomorphism

$$
\mathcal{J}^{s} \cong I_{\mathfrak{A}}\left(n / d_{2 s-1}, n / d_{2 s}\right) .
$$

(3) The inclusion of $J_{K}^{j}$ in $J^{j}$ identifies $\mathcal{J}_{K}^{j}$ with the set of $K^{\times}$-fixed points in $\mathcal{J}^{j}$. Setting $\mathcal{K}^{j}=$ $\mathcal{K} \cap \mathcal{J}^{j}$, we have

$$
\mathcal{J}^{j}=\mathcal{J}_{K}^{j} \perp \mathcal{K}^{j}, \quad \mathcal{K}=\bigoplus_{j} \mathcal{K}^{j} .
$$

Proof. The first and last assertions are immediate. The second follows from [BH05b, $\S 6.3]$.

As in $\S 1.2$, let $\boldsymbol{i}^{+}$be the greatest odd jump of $\beta$ over $F$, and $\boldsymbol{d}^{+}=d_{\boldsymbol{i}^{+}}$. In particular, all jumps of $\beta$ greater than $\boldsymbol{i}^{+}$are even. If, on the other hand, $2 s<\boldsymbol{i}^{+}$is a jump of $\beta$, we have $\mathcal{J}^{s}=\mathcal{J}_{K}^{s}$. We need therefore only ever consider even jumps greater than $\boldsymbol{i}^{+}$.

From part (2) of Lemma 7.2 we get

$$
\bigoplus_{2 s>\boldsymbol{i}^{+}} \mathcal{J}^{s} \cong I_{\mathbb{k}_{F}}\left(\Gamma ; n / \boldsymbol{d}^{+}, n\right)
$$




\section{TAME LANGLANDS CORRESPONDENCE: TOTALLY RAMIFIED REPRESENTATIONS}

Therefore we obtain the next proposition.

Proposition 7.2. The $\Gamma$-space $\mathcal{K}$ is isomorphic to the space of $x \in I_{\mathbb{k}_{F}}\left(\Gamma ; n / \boldsymbol{d}^{+}, n\right)$ on which the subgroup $\Gamma_{2}$ of $\Gamma$ of order 2 acts via its non-trivial character.

7.3 In this section we assume that $m=n / 2$ is odd. Thus $\Gamma_{2}$ is the 2 -Sylow subgroup $\Delta$ of $\Gamma$, and $\Delta$ acts on $\mathcal{K}$ via its non-trivial character. It follows that $\Delta$ has only the trivial fixed point on $\mathcal{K}$. By Proposition 4.7, therefore,

$$
\epsilon_{F} \epsilon_{K}=t_{\Gamma}(\mathcal{K})=t_{\Delta}(\mathcal{K}) .
$$

Proposition 7.3. Suppose that $m=n / 2$ is odd. Then

$$
\epsilon_{F} \epsilon_{K}=\left(\frac{-1}{q}\right)^{\left(\boldsymbol{d}^{+}-1\right) / 2} .
$$

Proof. From Proposition 7.2, we have $\operatorname{dim}_{\mathbb{k}_{F}} \mathcal{K}=m-m / \boldsymbol{d}^{+}$. Writing $q=\left|\mathbb{k}_{F}\right|=p^{f}$, the symplectic $\mathbb{F}_{p} \Delta$-module $\mathcal{K}$ is isometric to $H(U)$, where $U$ has $\mathbb{F}_{p^{-}}$dimension $f m\left(\boldsymbol{d}^{+}-1\right) / 2 \boldsymbol{d}^{+}=\delta$, say, and $\Delta$ acts on $U$ via its non-trivial character. Therefore

$$
t_{\Delta}(\mathcal{K})=(-1)^{\left(p^{\delta}-1\right) / 2} .
$$

However, $\boldsymbol{d}^{+}$is odd in this case and therefore $m\left(\boldsymbol{d}^{+}-1\right) / 2 \boldsymbol{d}^{+} \equiv\left(\boldsymbol{d}^{+}-1\right) / 2(\bmod 2)$. So

$$
\left(p^{\delta}-1\right) / 2 \equiv\left(q^{\left(\boldsymbol{d}^{+}-1\right) / 2}-1\right) / 2 \quad(\bmod 2)
$$

and

as required.

$$
t_{\Delta}(\mathcal{K})=\left(\frac{-1}{q}\right)^{\left(\boldsymbol{d}^{+}-1\right) / 2}
$$

7.4 We turn to the case where $m=n / 2$ is even. We recall $(\S 1.2)$ that $\boldsymbol{i}_{+}$is the least jump $j$ of $\beta$ (over $F$ ) such that $d_{j}$ is odd. Thus $\boldsymbol{i}_{+} \geqslant \boldsymbol{i}^{+}$, with equality if and only if $\boldsymbol{i}_{+}$is odd.

Proposition 7.4. Suppose that $d=2$ and that $m=n / 2$ is even. Then

$$
\epsilon_{F} \epsilon_{K}= \begin{cases}1 & \text { if } \boldsymbol{i}_{+} \text {is odd, } \\ \left(\frac{-1}{q}\right)^{n / 4}\left(\frac{2}{q}\right) & \text { if } \boldsymbol{i}_{+} \text {is even. }\end{cases}
$$

Proof. Again let $\Delta$ denote the 2-Sylow subgroup of $\Gamma$ and $\Gamma_{2}$ its subgroup of order 2. The group $\Gamma_{2}$ acts on $\mathcal{K}$ with only the trivial fixed point, hence the same applies to $\Delta$ and therefore

$$
\epsilon_{F} \epsilon_{K}=t_{\Delta}(\mathcal{K})
$$

Let $2^{a}$ be the exact power of 2 dividing $n$.

We first treat the case where $\boldsymbol{i}_{+}$is odd. It follows that $\boldsymbol{d}^{+}$is odd. The $\mathbb{k}_{F}$-dimension of $\mathcal{K}$ is $m-m / \boldsymbol{d}^{+}$, which is divisible by $2^{a}$. Thus, as $\mathbb{F}_{p} \Delta$-module, $\mathcal{K}$ is isomorphic to a direct sum of an even number of copies of the symplectic module $I_{\mathbb{F}_{p}}\left(\Delta ; 2^{a-1}, 2^{a}\right)$, whence $t_{\Delta}(\mathcal{K})=+1$, as required.

We therefore assume that $\boldsymbol{i}_{+}$is even, and so $\boldsymbol{i}_{+}>\boldsymbol{i}^{+}$. Thus $\boldsymbol{d}^{+}$is even. By definition, $\boldsymbol{i}_{+}$is the least jump $j$ of $\beta$ such that $K \not \subset E_{j}$. Therefore, if $2 s<\boldsymbol{i}_{+}$is a jump of $\beta$, we have $\mathcal{J}^{s}=\mathcal{J}_{K}^{s}$ and so

$$
\mathcal{K}=I_{\mathbb{k}_{F}}\left(\Gamma ; n / \boldsymbol{d}_{+}, n\right) \cap I_{\mathbb{k}_{F}}(\Gamma ; n / 2, n),
$$

where $\boldsymbol{d}_{+}=d_{\boldsymbol{i}_{+}-1}$. Both $\boldsymbol{d}_{+}$and $n / \boldsymbol{d}_{+}$are even, so

$$
I_{\mathbb{k}_{F}}\left(\Gamma ; n / \boldsymbol{d}_{+}, n\right)=\mathcal{K} \oplus I_{\mathbb{k}_{F}}\left(\Gamma ; n / \boldsymbol{d}_{+}, n / 2\right) .
$$




\section{J. Bushnell and G. Henniart}

Therefore $\mathcal{K}$ has dimension $n / 2$ and, as $\mathbb{k}_{F} \Delta$-module, it is isomorphic to the sum of an odd number of copies of $I_{\mathbb{k}_{F}}\left(\Delta ; 2^{a-1}, 2^{a}\right)$. The result therefore follows from the next lemma.

Lemma 7.4. Let $\Delta$ be cyclic of order $2^{a}, a \geqslant 2$. Then

$$
t_{\Delta}\left(I_{\mathbb{F}_{p}}\left(\Delta ; 2^{a-1}, 2^{a}\right)\right)=\left(\frac{-1}{p}\right)^{2^{a-2}}\left(\frac{2}{p}\right) .
$$

Proof. We abbreviate $I_{2}(\Delta)=I_{\mathbb{F}_{p}}\left(\Delta ; 2^{a-1}, 2^{a}\right)$.

Suppose first that $p \equiv 1(\bmod 4)$. In this case, we observe, $\Delta$ has no anisotropic (i.e., of ntsd type) symplectic modules over $\mathbb{F}_{p}$. For, let $\mathbb{k}=\mathbb{F}_{p^{r}}$ for some $r$ and let $\mathbb{k}^{\prime} / \mathbb{k}$ be the quadratic extension. The kernel $\mathcal{T}$ of the norm from $\mathbb{k}^{\prime}$ to $\mathbb{k}$ has order $p^{r}+1 \equiv 2(\bmod 4)$. It therefore has no subgroup of order divisible by 4 , so any character $\Delta \rightarrow \mathcal{T}$ has image contained in $\{ \pm 1\}$, and this cannot generate $\mathbb{k}^{\prime}$ as $\mathbb{F}_{p}$-algebra.

In this case, therefore, $I_{2}(\Delta)$ is hyperbolic, and a maximal totally isotropic subspace has dimension $2^{a-2}$ over $\mathbb{F}_{p}$. The required sign is therefore

$$
t_{\Delta}\left(I_{2}(\Delta)\right)=(-1)^{r(a)}, \quad r(a)=\left(p^{2^{a-2}}-1\right) / 2^{a} .
$$

An elementary inductive argument shows that

$$
\left(p^{2^{a-2}}-1\right) / 2^{a} \equiv(p-1) / 4 \quad(\bmod 2), \quad a \geqslant 2, p \equiv 1 \quad(\bmod 4) .
$$

Therefore

as required.

$$
t_{\Delta}\left(I_{2}(\Delta)\right)=(-1)^{(p-1) / 4}=\left(\frac{2}{p}\right) \quad \text { for } p \equiv 1 \quad(\bmod 4)
$$

We now assume that $p \equiv 3(\bmod 4)$. The simplest way to proceed is to construct a table. Let $\mathbb{k}_{j}$ denote the field of $p^{2^{j}}$ elements.

Suppose first that $p \equiv 3(\bmod 8)$. The field obtained by adjoining to $\mathbb{F}_{p}$ a primitive $2^{a}$-root of unity is $\mathbb{k}_{1}$ for $a=2$ or 3 and $\mathbb{k}_{a-2}$ for $a \geqslant 4$. Thus $I_{2}(\Delta)$ is the anisotropic space $\mathbb{k}_{1}$ when $a=2$ (giving $t=+1$ ), the hyperbolic space $\mathbb{k}_{1} \oplus \mathbb{k}_{1}$ when $a=3$ (giving $t=-1$ ) and the hyperbolic space $\mathbb{k}_{a-2} \oplus \mathbb{k}_{a-2}$ when $a \geqslant 4$ (giving $t=-1$ ), all as required.

Suppose now that $p \equiv 7(\bmod 8)$. There is an integer $b \geqslant 4$ such that the field of $2^{a}$ roots of unity over $\mathbb{F}_{p}$ is $\mathbb{k}_{1}$ for $2 \leqslant a \leqslant b$, and $\mathbb{k}_{a-b+1}$ for $a>b$. The space $I_{2}(\Delta)$ is therefore the anisotropic space $\mathbb{k}_{1}$ when $a=2$ (giving $t=-1$ ), the sum of two anisotropic spaces $\mathbb{k}_{1}$ when $a=3$ (giving $t=+1$ ), and the sum of four copies of $\mathbb{k}_{1}$ when $a=4$ (giving $t=+1$ ). For $a \geqslant 5, I_{2}(\Delta$ ) is a sum of $4 r$ copies of some field, for some integer $r$, giving $t=+1$. Thus Lemma 7.4 is proved.

This completes the proof of Proposition 7.4.

\section{Gauss sums}

In this section, we calculate the quotient $\mathfrak{G}_{F}\left(\varpi, \psi_{F}\right) / \mathfrak{G}_{K}\left(\varpi, \psi_{K}\right)$ of products of Gauss sums appearing in Theorem 6.6. Throughout, we assume that $d=[K: F]=2$.

We will so prove Theorems 3.1 and 3.2 in all cases except that where $\boldsymbol{i}_{+}=\boldsymbol{i}^{+}=r_{1}=1$ (in the notation of $\S 1.2)$.

8.1 By definition (5.7.4), we have

$$
\mathfrak{G}_{F}\left(\varpi, \psi_{F}\right)=\prod_{i \in S} \mathfrak{g}\left(\zeta_{i}(\varpi) \boldsymbol{q}_{F}^{(i)}, \psi_{F}\right),
$$




\section{TAME LANGLANDS CORRESPONDENCE: TOTALLY RAMIFIED REPRESENTATIONS}

where, we recall, $S$ is the set of integers $i$ such that $r_{i}$ is an odd jump of $\beta$ with $r_{i} \geqslant 3$. Similarly,

$$
\mathfrak{G}_{K}\left(\varpi, \psi_{K}\right)=\prod_{i \in S} \mathfrak{g}\left(\zeta_{i}(\varpi) \boldsymbol{q}_{K}^{(i)}, \psi_{K}\right),
$$

with the understanding that $\mathfrak{g}\left(\zeta_{i}(\varpi) \boldsymbol{q}_{K}^{(i)}, \psi_{K}\right)=1$ if $r_{i}$ is not a jump of $\beta$ relative to $K$.

LEMMA 8.1.

(1) If $\boldsymbol{i}^{+}=1$, then $\mathfrak{G}_{F}\left(\varpi, \psi_{F}\right)=\mathfrak{G}_{K}\left(\varpi, \psi_{K}\right)=1$ and $S$ is empty.

(2) If $i \in S$ satisfies $r_{i}<\boldsymbol{i}^{+}$, we have $\mathcal{M}_{i}=\mathcal{M}_{K, i}$ and $\mathfrak{g}\left(\zeta_{i}(\varpi) \boldsymbol{q}_{F}^{(i)}, \psi_{F}\right)=\mathfrak{g}\left(\zeta_{i}(\varpi) \boldsymbol{q}_{K}^{(i)}, \psi_{K}\right)$.

(3) If $r_{1}=1$ and $K \subset E_{1}$, then $\mathfrak{K}_{F}\left(\alpha_{1}, \varpi, \psi_{F}\right)=\mathfrak{K}_{F}^{\varkappa}\left(\alpha_{1}, \varpi, \psi_{F}\right)$.

Proof. The first two assertions follow directly from the definitions. In part (3), the character $\varkappa$ odet is trivial on the $G$-centralizer of $E_{1}$ and, in particular, on $\boldsymbol{\mu}_{1}$. The assertion follows from the definitions in $\S 6.6$.

The hypothesis of part (3) of Lemma 8.1 holds if either $\boldsymbol{i}^{+}>r_{1}=1$ or $\boldsymbol{i}_{+}>\boldsymbol{i}^{+} \geqslant r_{1}=1$. Consequently, we have the following proposition.

Proposition 8.1. Suppose that $r_{1}=1$ and that $\mathfrak{K}_{F}^{\varkappa}\left(\alpha_{1}, \varpi, \psi_{F}\right) \neq \mathfrak{K}_{F}\left(\alpha_{1}, \varpi, \psi_{F}\right)$. Then $\boldsymbol{i}_{+}=\boldsymbol{i}^{+}=$ $r_{1}=1$.

We can now simplify our notation: if $r_{j}=\boldsymbol{i}^{+}>1$, we set $\zeta_{j}(\varpi)=\zeta(\varpi), \boldsymbol{q}_{F}^{(j)}=\boldsymbol{q}_{F}$. Therefore

$$
\frac{\mathfrak{G}_{F}\left(\varpi, \psi_{F}\right)}{\mathfrak{G}_{K}\left(\varpi, \psi_{K}\right)}=\frac{\mathfrak{g}\left(\zeta(\varpi) \boldsymbol{q}_{F}, \psi_{F}\right)}{\mathfrak{g}\left(\zeta(\varpi) \boldsymbol{q}_{K}, \psi_{K}\right)}
$$

8.2 We assume from now on that $\boldsymbol{i}^{+}>1$. Let us write $\mathcal{M}_{0}$ rather than $\mathcal{M}_{\boldsymbol{i}^{+}}$. With $\Gamma=E^{\times} / F^{\times} U_{E}^{1}$ as before, $\mathcal{M}_{0}$ is $\Gamma$-isomorphic to the $\mathbb{k}_{F} \Gamma$-submodule $I_{\mathbb{k}_{F}}\left(\Gamma ; n / \boldsymbol{d}^{\prime}, n / \boldsymbol{d}^{+}\right)$of $\mathbb{k}_{F} \Gamma$, where $\boldsymbol{d}^{\prime}=d_{\boldsymbol{i}^{+}-1}$.

To describe the quadratic form $\boldsymbol{q}_{F}$, we compare with the objects $Q_{\Gamma}, \varepsilon_{\Gamma}$ introduced in $\S 4.6$, via an isomorphism $\mathfrak{A} / \mathfrak{P} \cong \mathbb{k}_{F} \Gamma$ as in $\S 4.2$. We let $\gamma$ denote the generator $x \mapsto \varpi^{-1} x \varpi$ of $\Gamma$. We write $\boldsymbol{i}^{+}=1+2 s$. The form $\boldsymbol{q}_{F}$ is the restriction to $\mathcal{M}_{0}$ of $x \mapsto \varepsilon_{\Gamma}\left((x-\gamma x)\left(\gamma^{s} \bar{x}\right)\right)$.

There is a unique $\Gamma$-submodule $\mathcal{Y}$ of $\mathcal{M}_{0}$ such that $\mathcal{M}_{0}=\mathcal{M}_{0, K} \oplus \mathcal{Y}$. The functions $\psi_{F} \circ \boldsymbol{q}_{F}$ and $\psi_{K} \circ \boldsymbol{q}_{K}$ agree on $\mathcal{M}_{0, K}$, so

$$
\frac{\mathfrak{G}_{F}\left(\varpi, \psi_{F}\right)}{\mathfrak{G}_{K}\left(\varpi, \psi_{K}\right)}=\mathfrak{g}\left(\zeta(\varpi) \boldsymbol{q}_{F} \mid \mathcal{Y}, \psi_{F}\right)
$$

8.3 We start with the following special case.

Proposition 8.3. Suppose that $\boldsymbol{i}^{+}>1$ and that $m=n / 2$ is odd. The quadratic form $\boldsymbol{q}_{F} \mid \mathcal{Y}$ is non-degenerate and has discriminant $(-1)^{s} \boldsymbol{d}^{+}$.

Proof. In the notation of $\S 4.1, \mathcal{M}_{0} \cong I_{\mathbb{k}_{F}}\left(\Gamma ; n / \boldsymbol{d}^{\prime}, n / \boldsymbol{d}^{+}\right)$is the direct sum of the spaces $V_{\chi}$, where $\chi$ ranges over those elements of $\Omega \backslash \widehat{\Gamma}$ such that $\chi^{n / d^{+}}=1 \neq \chi^{n / d^{\prime}}$. The space $\mathcal{Y}$ is the sum of those $V_{\chi}$ for which $\chi^{n / 2} \neq 1$. Since $\boldsymbol{d}^{+}$is odd, we have $\mathcal{Y} \cong I_{\mathbb{k}_{F}}\left(\Gamma ; n / 2 \boldsymbol{d}^{+}, n / \boldsymbol{d}^{+}\right)$. In particular

$$
\operatorname{dim}_{\mathbb{k}_{F}} \mathcal{Y}=m / \boldsymbol{d}^{+} \text {. }
$$

Let $\mathbb{k} / \mathbb{k}_{F}$ be a finite field extension. We can extend $\boldsymbol{q}_{F}$, by linearity, to a quadratic form $\boldsymbol{q}_{F, \mathbb{k}}$ : $\mathcal{Y} \otimes \mathbb{k} \rightarrow \mathbb{k}$. The form $\boldsymbol{q}_{F}$ is then non-degenerate on $\mathcal{Y}$ if and only if $\boldsymbol{q}_{F, \mathbb{k}}$ is non-degenerate on $\mathcal{Y} \otimes \mathbb{k}$. In other words, to prove non-degeneracy, we can proceed as if all characters of $\Gamma$ take their values in $\mathbb{k}_{F}^{\times}$. 


\section{J. Bushnell and G. Henniart}

For a character $\chi$ of $\Gamma$, let

$$
e_{\chi}=n^{-1} \sum_{\gamma \in \Gamma} \chi(\gamma) \gamma^{-1}
$$

denote the corresponding idempotent of $\mathbb{k}_{F} \Gamma$. Thus $V_{\chi}=e_{\chi} \mathbb{k}_{F} \Gamma$. Consider first the case where $\chi$ has order 2. Then $V_{\chi} \subset \mathcal{Y}$ and

$$
\boldsymbol{q}_{F}\left(e_{\chi}\right)=(-1)^{s} 2 \varepsilon\left(e_{\chi} \bar{e}_{\chi}\right)=(-1)^{s} 2 \varepsilon\left(e_{\chi}\right)=(-1)^{s} 2 / n .
$$

In particular, $\boldsymbol{q}_{F} \mid V_{\chi}$ is non-degenerate in this case.

Now let $V_{\chi} \subset \mathcal{Y}, \chi \neq \chi^{-1}$, and consider the restriction of $\boldsymbol{q}_{F}$ to $V_{\chi} \oplus V_{\chi^{-1}}$. We set $e_{\chi}=e$ (so that $\left.\bar{e}=e_{\chi^{-1}}\right)$ and $\alpha=\chi(\gamma)$ :

$$
\begin{aligned}
\boldsymbol{q}_{F}(x e+y \bar{e}) & =\varepsilon\left((x e+y \bar{e})\left(\alpha^{s} x \bar{e}+\alpha^{-s} e\right)-\left(\alpha x e+\alpha^{-1} y \bar{e}\right)\left(\alpha^{s} x \bar{e}+\alpha^{-s} e\right)\right) \\
& =x y\left(\alpha^{-s}+\alpha^{s}-\alpha^{1-s}-\alpha^{s-1}\right) / n .
\end{aligned}
$$

This vanishes identically if and only if $\alpha=1$ or $\alpha^{2 s+1}=1$; since $\alpha$ is a root of unity of even order, we conclude that $\boldsymbol{q}_{F}$ is non-degenerate on $V_{\chi} \oplus V_{\chi^{-1}}$. Thus $\boldsymbol{q}_{F}$ is non-degenerate, as desired.

We return to the given base field $\mathbb{k}_{F}$. If $\chi_{2}$ denotes the character of $\Gamma$ of order 2 , the calculation above shows that $\boldsymbol{q}_{F} \mid V_{\chi_{2}}$ has discriminant $(-1)^{s} 2 / n$, while $Q_{\Gamma} \mid V_{\chi_{2}}$ has discriminant $1 / n$. We conclude (Proposition 4.4, Remark 4.5) that the discriminants of $\boldsymbol{q}_{F}\left|\mathcal{Y}, Q_{\Gamma}\right| \mathcal{Y}$ differ by a factor of $(-1)^{s} 2$.

We are therefore reduced to calculating the discriminant of $Q_{\Gamma}$ on $\mathcal{Y}$. Its discriminant on $\mathbb{k}_{F}\left[\Gamma / \Gamma^{n / \boldsymbol{d}^{+}}\right]$is $\left(\boldsymbol{d}^{+}\right)^{n / \boldsymbol{d}^{+}}=1$, while on $\mathbb{k}_{F}\left[\Gamma / \Gamma^{n / 2 \boldsymbol{d}^{+}}\right]$its discriminant is $\left(2 \boldsymbol{d}^{+}\right)^{n / 2 \boldsymbol{d}^{+}}=2 \boldsymbol{d}^{+}$ (cf. (4.6.1)). The discriminant of $Q_{\Gamma} \mid \mathcal{Y}$ is therefore $2 \boldsymbol{d}^{+}$, whence the discriminant of $\boldsymbol{q}_{F}$ on $\mathcal{Y}$ is $(-1)^{s} \boldsymbol{d}^{+}\left(\bmod \mathbb{k}_{F}^{\times 2}\right)$, as required.

The $\mathbb{k}_{F}$-dimension of $\mathcal{Y}$ is $m / \boldsymbol{d}^{+}$, which is odd, so we have the corollary.

Corollary 8.3. In the case $n / 2$ odd and $\boldsymbol{i}^{+}>1$, we have

$$
\frac{\mathfrak{G}_{F}\left(\varpi, \psi_{F}\right)}{\mathfrak{G}_{K}\left(\varpi, \psi_{K}\right)}=\left(\frac{-1}{q}\right)^{\left(\boldsymbol{i}^{+}-1\right) / 2}\left(\frac{\boldsymbol{d}^{+}}{q}\right)\left(\frac{\zeta(\varpi)}{q}\right) \mathfrak{g}\left(\psi_{F}\right)^{m / \boldsymbol{d}^{+}} .
$$

8.4 We now consider the case where $d=2$ and $n / 2$ is even.

Proposition 8.4. Suppose that $n / 2$ is even and $\boldsymbol{i}^{+}>1$. Then

$$
\frac{\mathfrak{G}_{F}\left(\varpi, \psi_{F}\right)}{\mathfrak{G}_{K}\left(\varpi, \psi_{K}\right)}= \begin{cases}1 & \text { if } \boldsymbol{i}_{+} \text {is even, } \\ \left(\frac{-1}{q}\right) & \text { if } \boldsymbol{i}_{+} \text {is odd. }\end{cases}
$$

Proof. In the first case, where $\boldsymbol{i}_{+}$is even, all factors in the definitions of the Gauss sums are identically equal, and the result is immediate.

Thus we assume $\boldsymbol{i}_{+}=\boldsymbol{i}^{+}$is odd; it follows that $\boldsymbol{d}^{+}$is odd. In this case, we have $\mathcal{Y} \cong I_{\mathbb{k}_{F}}\left(\Gamma ; n / 2 \boldsymbol{d}^{+}\right.$, $n / \boldsymbol{d}^{+}$). From (8.2.1), we have to show that $\boldsymbol{q}_{F} \mid \mathcal{Y}$ is non-degenerate and then compute its discriminant. Since $n / 2 \boldsymbol{d}^{+}$is even, the quadratic character of $\Gamma$ does not occur in $\mathcal{Y}$. The proof of nondegeneracy is then the same as in $\S 8$. Further, $\boldsymbol{q}_{F}$ and $Q_{\Gamma}$ have the same discriminant on $\mathcal{Y}$ $(\S 4.4)$. It follows from (4.6.2) that this discriminant is 1 . The $\mathbb{k}_{F}$-dimension of $\mathcal{Y}$ is $n / 2 \boldsymbol{d}^{+}$, so

$$
\mathfrak{G}_{F}\left(\varpi, \psi_{F}\right) / \mathfrak{G}_{K}\left(\varpi, \psi_{K}\right)=\mathfrak{g}\left(\psi_{F}\right)^{n / 2 \boldsymbol{d}^{+}}=\left(\frac{-1}{q}\right)^{n / 4},
$$

as required. 


\section{TAME LANGLANDS CORRESPONDENCE: TOTALLY RAMIFIED REPRESENTATIONS}

8.5 Let us prove Theorem 3.1 in the case $\boldsymbol{i}^{+}>1$. Thus $n / 2$ is odd. The factor $\epsilon_{F} \epsilon_{K}$ in Theorem 6.6 is given by Proposition 7.3. In the case $r_{1}=1$, the factor $\mathfrak{K}_{F}^{\varkappa}\left(\alpha_{1}, \varpi, \psi_{F}\right) / \mathfrak{K}_{F}\left(\alpha_{1}, \varpi, \psi_{F}\right)$ is 1 , by Proposition 8.1. The result now follows from Corollary 8.3.

We turn to Theorem 3.2. In particular, $n / 2$ is even. Excepting the case $\boldsymbol{i}_{+}=\boldsymbol{i}^{+}=r_{1}=1$, the result follows from Propositions 8.4 and 7.4.

\section{Kloosterman sums}

To complete the proofs of Theorems 3.1 and 3.2 , it remains only to treat the cases where $\boldsymbol{i}_{+}=\boldsymbol{i}^{+}=$ $r_{1}=1$. In particular, we have $K \cap E_{1}=F$.

9.1 In our present situation, Theorem 6.6 reduces to

$$
\mu(\varpi)=\epsilon_{F} \epsilon_{K} \frac{\mathfrak{K}_{F}^{\varkappa}\left(\alpha_{1}, \varpi, \psi_{F}\right)}{\mathfrak{K}_{F}\left(\alpha_{1}, \varpi, \psi_{F}\right)} k,
$$

for some positive constant $k$. The sign $\epsilon_{F} \epsilon_{K}$ is given by Proposition 7.3 (when $m=n / 2$ is odd) or the first case of Proposition 7.4 when $m=n / 2$ is even.

We note that, in the present situation, we have $\zeta(\varpi)=\alpha_{1} \varpi$.

9.2 We treat first a special case. We assume that $E_{1}=F$ and $\beta=\alpha_{1}$. In particular, $\beta^{-1}$ is a prime element of $E$. As before, we set $\pi={ }_{F} \pi_{\mu \xi}=\mathrm{A}_{K / F} \rho, \rho={ }_{K} \pi_{\xi}$.

We consider the relation between the Godement-Jacquet local constants (see [GJ72] and [Jac79]) $\varepsilon\left(\pi, s, \psi_{F}\right)$ and $\varepsilon\left(\rho, s, \psi_{K}\right)$, where $\psi_{K}=\psi_{F} \circ \operatorname{Tr}_{K / F}$. Since $\beta$ has no even jumps in this case, we have $\epsilon_{F}=\epsilon_{K}=1$ and $\pi=c$-Ind $\Lambda$, for a quasicharacter $\Lambda$ of $\boldsymbol{J}$ with $\Lambda \mid E^{\times}=\mu \xi$. We use the technique of [BF85] and [Bus87] (or see [Bus91] for a convenient summary); this approach gives

$$
\varepsilon\left(\pi, \frac{1}{2}, \psi_{F}\right)=k_{1} \mu \xi(\beta)^{-1} \psi_{F}\left(\operatorname{Tr}_{E / F}(\beta)\right),
$$

for some $k_{1}>0$. Similarly,

$$
\varepsilon\left(\rho, \frac{1}{2}, \psi_{K}\right)=k_{2} \xi(\beta)^{-1} \psi_{F}\left(\operatorname{Tr}_{E / F}(\beta)\right),
$$

for some $k_{2}>0$. However, the relation $\pi=\mathrm{A}_{K / F} \rho$ gives [HH95] and [HL05],

$$
\varepsilon\left(\pi, \frac{1}{2}, \psi_{F}\right)=\varepsilon\left(\rho, \frac{1}{2}, \psi_{K}\right) \lambda_{K / F}^{m}
$$

whence, by Lemma 1.5, $\mu(\beta)=k_{3} \mathfrak{g}\left(\psi_{F}\right)^{-m}$, for some $k_{3}>0$. Combining with (9.1.1), we therefore have, for various $k>0$ and any prime element $\varpi$ of $E$,

$$
\frac{\mathfrak{K}_{F}^{\varkappa}\left(\alpha_{1}, \varpi, \psi_{F}\right)}{\mathfrak{K}_{F}\left(\alpha_{1}, \varpi, \psi_{F}\right)}= \begin{cases}k\left(\frac{\alpha_{1} \varpi}{q}\right) \mathfrak{g}\left(\psi_{F}\right)^{m} & \text { if } m \text { is odd, } \\ k\left(\frac{-1}{q}\right)^{m / 2} & \text { if } m \text { is even. }\end{cases}
$$

9.3 We revert to the general case, as at the beginning of the section. Writing $\varkappa_{1}=\varkappa \circ \mathrm{N}_{E_{1} / F}$, the definitions of $\S 6.6$ give

$$
\mathfrak{K}_{F}\left(\alpha_{1}, \varpi, \psi_{F}\right)=\mathfrak{K}_{E_{1}}\left(\alpha_{1}, \varpi, \psi_{E_{1}}\right), \quad \mathfrak{K}_{F}^{\varkappa}\left(\alpha_{1}, \varpi, \psi_{F}\right)=\mathfrak{K}_{E_{1}}^{\varkappa_{1}}\left(\alpha_{1}, \varpi, \psi_{E_{1}}\right) .
$$

We have $\left[E_{1}: F\right]=\boldsymbol{d}^{+} \equiv 1(\bmod 2)$ and $\psi_{E_{1}}\left|\mathbb{k}_{F}=\psi_{F}^{\boldsymbol{d}^{+}}\right| \mathbb{k}_{F}$. If $m$ is odd, (9.2.1) therefore says

$$
\frac{\mathfrak{K}_{F}^{\varkappa}\left(\alpha_{1}, \varpi, \psi_{F}\right)}{\mathfrak{K}_{F}\left(\alpha_{1}, \varpi, \psi_{F}\right)}=k\left(\frac{\boldsymbol{d}^{+}}{q}\right)\left(\frac{\zeta(\varpi)}{q}\right) \mathfrak{g}\left(\psi_{F}\right)^{m / \boldsymbol{d}^{+}},
$$

exactly as required for Theorem 3.1 . 


\section{J. Bushnell and G. Henniart}

In the case where $m$ is even, we get

$$
\frac{\mathfrak{K}_{F}^{\varkappa}\left(\alpha_{1}, \varpi, \psi_{F}\right)}{\mathfrak{K}_{F}\left(\alpha_{1}, \varpi, \psi_{F}\right)}=k\left(\frac{-1}{q}\right)^{n / 4},
$$

as required for Theorem 3.2.

We have now completed the proofs of Theorems 3.1 and 3.2 .

\section{REFERENCES}

AC89 J. Arthur and L. Clozel, Simple algebras, base change, and the advanced theory of the trace formula, Annals of Mathematics Studies, vol. 120 (Princeton University Press, Princeton, NJ, 1989).

BF83 C. J. Bushnell and A. Fröhlich, Gauss sums and p-adic division algebras, Lecture Notes in Mathematics, vol. 987 (Springer, Berlin, 1983).

BF85 C. J. Bushnell and A. Fröhlich, Non-abelian congruence Gauss sums and p-adic simple algebras, Proc. London Math. Soc. (3) 50 (1985), 207-264.

BH96 C. J. Bushnell and G. Henniart, Local tame lifting for GL(N) I: simple characters, Publ. Math. Inst. Hautes Études Sci. 83 (1996), 105-233.

BH05a C. J. Bushnell and G. Henniart, The essentially tame local Langlands correspondence, I, J. Amer. Math. Soc., to appear.

BH05b C. J. Bushnell and G. Henniart, Local tame lifting for $\mathrm{GL}_{n}$, III: explicit base change and JacquetLanglands correspondence, J. reine angew. Math. 580 (2005), 39-100.

BK93 C. J. Bushnell and P. C. Kutzko, The admissible dual of GL( $N)$ via compact open subgroups, Annals of Mathematics Studies, vol. 129 (Princeton University Press, Princeton, NJ, 1993).

Bus87 C. J. Bushnell, Hereditary orders, Gauss sums and supercuspidal representations of $\mathrm{GL}_{N}$, J. reine angew. Math. 375/376 (1987), 184-210.

Bus91 C. J. Bushnell, Gauss sums and local constants for $\mathrm{GL}(N)$, in L-functions and arithmetic, eds J. Coates and M. J. Taylor, London Mathematical Society Lecture Notes, vol. 153 (Cambridge University Press, Cambridge, 1991), 61-73.

Duk92 W. Duke, On multiple Salié sums, Proc. Amer. Math. Soc. 114 (1992), 623-625.

Gla68 G. Glauberman, Correspondences of characters for relatively prime operator groups, Canad. J. Math. 20 (1968), 1465-1488.

GJ72 R. Godement and H. Jacquet, Zeta functions of simple algebras, Lecture Notes in Mathematics, vol. 260 (Springer, Berlin, 1972).

Hen00 G. Henniart, Une preuve simple des conjectures locales de Langlands pour $\mathrm{GL}_{n}$ sur un corps p-adique, Invent. Math. 139 (2000), 439-455.

HH95 G. Henniart and R. Herb, Automorphic induction for GL(n) (over local non-archimedean fields), Duke Math. J. 78 (1995), 131-192.

HL05 G. Henniart and B. Lemaire, work in progress (2005).

How77 R. E. Howe, Tamely ramified supercuspidal representations of $\mathrm{GL}_{n}$, Pacific J. Math. 73 (1977), 437-460.

HT01 M. Harris and R. Taylor, On the geometry and cohomology of some simple Shimura varieties, Annals of Mathematics Studies, vol. 151 (Princeton University Press, Princeton, NJ, 2001).

Jac79 H. Jacquet, Principal L-functions of the linear group, in Automorphic forms, representations and L-functions, eds A. Borel and W. Casselman, Proc. Symp. Pure Mathematics, vol. 33, Part II (American Mathematical Society, Providence, RI, 1979), 63-86.

LRS93 G. Laumon, M. Rapoport and U. Stuhler, D-elliptic sheaves and the Langlands correspondence, Invent. Math. 113 (1993), 217-338.

Moy86 A. Moy, Local constants and the tame Langlands correspondence, Amer. J. Math. 108 (1986), 863-930. 


\section{TAME LANGLANDS CORRESPONDENCE: TOTALLY RAMIFIED REPRESENTATIONS}

Rei91 H. Reimann, Representations of tamely ramified p-adic division and matrix algebras, J. Number Theory 38 (1991), 58-105.

Sal32 H. Salié, Über die Kloostermanschen Summen S(u,v;q), Math. Z. 34 (1932), 91-109.

Tat79 J. T. Tate, Number-theoretic background, in Automorphic forms, representations and L-functions, eds A. Borel and W. Casselman, Proc. Symp. Pure Mathematics, vol. 33, Part II (American Mathematical Society, Providence, RI, 1979), 3-26.

Wil89 W. Willems, Representations of certain group extensions, in Representation theory and number theory in connection with the Local Langlands Conjecture, ed. J. Ritter, Contemporary Mathematics, vol. 86 (American Mathematical Society, Providence, RI, 1989), 91-99.

Colin J. Bushnell bushnell@mth.kcl.ac.uk

Department of Mathematics, King's College, Strand, London WC2R 2LS, UK

Guy Henniart Guy.Henniart@math.u-psud.fr

Département de Mathématiques et UMR 8628 du CNRS, Bâtiment 425, Université de Paris-Sud, 91405 Orsay cedex, France 